\title{
CHOIX ET FIABILITÉ D'UN MODĖLE DE RÉTROCALCUL DES TAILLES EN ÉCOLOGIE HALIEUTIQUE. MISE AU POINT D'UN LOGICIEL INFORMATIQUE
}

\author{
E. BAGLINIERE (1), Dominique OMBREDANE (2)
}

(1) Conseil Supérieur de la Pêche, Centre du Paraclet, BP 5, 80440 BOVES.

(2) Laboratoire d'Écologie Hydrobiologique, INRA, 65, rue de St-Brieuc, 35042 RENNES Cédex.

\section{RÉSUMÉ}

Les modèles de rétrocalcul des tailles à partir de mesures de pièces osseuses sont souvent utilisés sans précautions en dynamique des populations et en écologie halieutiques. Le choix d'un modèle doit être justifié et sa validité doit être estimée. ll a doncété développé un logiciel informatique qui permet l'ajustement des différents modèles proposés par la littérature (linéarisables et polynomiaux) et le rétrocalcul des tailles parplusieurs méthodes. Celogiciel donne aussi des critères pour sélectionner le meilleur modèle et évaluer sa fiabilité: graphes, tests permettant d'analyser la "représentativité" d'une régression, ainsi que la normalité etl'indépendance de ses résidus, intervalle de confiance des tailles rétrocalculées.

Ce logiciel est testé sur une population (239 individus) de juvéniles migrants de Truite de mer (Salmo trutta $L$ ) piégés sur une rivière de Normandie ainsi que sur un sous-échantillon issu de cette population.

\section{CHOICE AND ACCURACY OF BACK - CALCULATING LENGTHS MODEL IN FISHERIES ECOLOGY. DEVELOPMENT OF A COMPUTER PROGRAM}

\section{SUMMARY}

Models to back-calculate lengths measurements from hard parts are often used without precautions in studies of population dynamics and fisheries ecology. The choice of a model must be justified and its validity has to be evaluated. A computer program is proposed to fit most of the models found in literature (linearizable, polynomial) and to back-calculate the lengths according to different methods. Some criteria to choose the best model and to estimate its reliability are given: scatter plots, statistical tests to check the model's significance and to evaluate the normality and independance properties of the residuals, the confidence limits for back-calculating lengths.

The program is first checked on a 239 young migrating sea trout (Salmo trutta L.) trapped in a Normandy river and then on a subset of the total sample.

\section{INTRODUCTION}

En biologie halieutique, le rétrocalcul des tailles à partir des mesures de pièces osseuses pour des études de croissance est une pratique très ancienne (LEA, 1910 ; LEE, 1912 in BEALL, 1979, ...). Le principe général est d'abord d'ajuster une relation $Y=f(X)$ entre la longueur $Y$ du poisson et la taille $X$ d'une pièce osseuse (écaille, otolithe, opercule,...). Puis le modèle est utilisé, avec ou sans transformation, dans un but "prédictif" pour calculer les longueurs $Y_{j}$, qu'avaient les poissons à des périodes "remarquables" j de leur vie, inscrites dans leurs pièces osseuses (mensuration $X_{i}$ ).

Depuis le modèle de "DAHL-LEA", $(Y=a X)$, traduisant une croissance isométrique des poissons, on voit apparaître dans la littératured'autres modèles $Y=f(X)$ dont une synthèse historique a été faite par HILE(1970). Dansl'abondante bibliographie concernant le sujet, on trouve divers essais d'amélioration des méthodes de rétrocalcul $Y_{i}=f(X, X, Y)$, et d'interprétation biologique des modèles (CARLANDER, 1981, 1982 et 1985). De plus, le développement des outils informatiques de calcul a permis d'ajuster des modèles de plus en plus complexes (CARLANDER, 1950; JONSSON et STENSETH, 1961), de tester leur fiabilité et de les comparer (JONSSON etSTENSETH, 1961;BEALL, 1979 ; ECHEVERRIA, 1987).

Cependant dans la pratique courante, beaucoup de travaux utilisent aveuglement les modèles proposés par la bibliographie pour l'espèce étudiée, sans d'autres précautions que quelques 
statistiques, nettement insuffisantes à notre avis pour évaluer l'adéquation du modèle. Par exemple, la plupart des modèles étant ajustés par régressions (linéaire, curvilinéaires ou polynomiales), de nombreux auteurs se contentent d'estimer le coefficient de détermination et de vérifier s'il est significativement différent de 0 . Pourtant, des biométriciens (TRANCHEFORT, 1974; TOMASSONNE et al, 1983...), conscients de l'utilisation "abusive" des régressions proposent des outils performants pour tester leur signification, leur validité, leur fiabilité et contrôler l'utilisation prédictive de tels modèles.

L'objet de notre travail est de faire le point sur la procédure à adopter pour choisir et utiliser un modèle de rétrocalcul. La démarche exposée n'est certes pas nouvelle dans le domaine de la biométrie, mais elle trouve son application dans un logiciel informatique que nous avons élaboré. S'il existe de nombreux programmes de régression dans le commerce, ils ne sont pas véritablement adaptés aux données que le biologiste des pêches doit traiter. Notamment, les échantillons étant constitués d'individus i appartenant à différentes cohortes, le nombre de mensurations $X_{i, j}$ (rayon de la pièce osseuse à l'âge j) n'est pas constant. Le fichier de données possède donc des lignes de longueur variable. FRIE (1982) a mis au point un programme BASICqui est utilisé en interactif avec un micromètre pour la saisie des rétromesures. Ce logiciel permet l'ajustement des modèles linéaires et curvilinénaires et calcule les tailles moyennes par âge et par classe d'âge. Cependant il ne propose que peu de critères permettant de valider le modèle ajusté. C'est pourquoi, nous avons mis au point un logiciel non seulement adapté aux données biologiques, mais assurant une certaine rigueur mathématique.

Ce logiciel ajuste tous les modèles linéaires, curvilinéaires et polynomiaux $Y=f(X)$, proposés par la littérature pour le rétrocalcul. Divers graphiques et tests statistiques permettent d'évaluer leur validité et de choisir le plus adéquat pour l'échantillon analysé. Le rétrocalcul des tailles $Y_{i, j}$ à partir des $X_{i, j}$, est effectué par différentes méthodes pour le modèle ajusté. Il peut, de plus, être réalisé par la méthode de DAHL-LEA et par celle de FRASER-LEE avec une valeur "standard" de la constante. La précision des résultats peut être estimée par l'intervalle de confiance autour des tailles retrocalculées. Ensuite sont estimées les tailles moyennes et leurvariance à chaqueâge pourles différentes cohortes (paramètres utiles en dynamique des populations). Enfin, comme la mensuration des pièces osseuses est un travail fastidieux, il est prévu une option permettant d'ajuster le modèle sur un sous échantillon et d'extrapoler par pondération, les résultats des rétrocalculs à l'ensemble de l'échantillon prélevé.

Les possibilités offertes par le logiciel sont illustrées à travers l'exemple d'une population de juvéniles migrants de Truite de mer.

\section{Choix du meilleur modèle $Y=f(X)$}

Le Tableau I résume les différents modèles proposés par la bibliographie, mettanten relation la longueur $Y$ des poissons et la mesure $X$ du plus grand rayon d'une pièce anatomique osseuse portant des annuli.

Ces modèles, ne sont en réalité que des cas particuliers d'un modèle polynomial de degrés $p$ $(p \geq 1)$ soit :

$Y=a_{0}+a_{1} X^{1}+a_{2} X^{2}+\ldots a_{p} X^{p}+\Sigma$

avec $\quad \hat{=}=$ termed'erreur

$\mathrm{a}_{\mathrm{o}}, \mathrm{a}_{1}, \mathrm{a}_{2} \ldots \mathrm{ap}=$ constantes du modèle.

Les modèles isométrique et linéaire sont des polynomes de degrés 1 ainsi que les modèles puissance et exponentiel après transformation logarithmique des variables $Y$ et (ou) $X$. le logiciel.

Parmi les modèles du Tableau I, seul celui de FRY (1943) n'a pu être pris en considérationdans

\subsection{Ajustement des modèles}

L'ajustement consiste en l'estimation des $p+1$ constantes $a_{0}, a_{1} \ldots$, ap à partir de $n$ couples de données expérimentales $\left(X_{i}, Y_{i}\right)$. Le principe de calcul du logiciel est basé sur la résolution d'un système de $n$ équations à $p+1$ inconnues (HAUT, 1982 ; LEBART et al, 1982) par le critère des moindres carrés. On minimisera donc la somme des carrés des écarts au modèle:

$$
\sum_{i=1}^{n}\left(Y_{i}-\hat{Y}_{i}\right)^{2}
$$

avec $Y_{i}=$ valeur observée

avec $\hat{Y}_{i}=$ valeur estimée par le modèle en fonction de $X_{i}$ et par rapport aux constantes à déterminer. Ce principe de calcul est utilisé pour l'ensemble des modèles ajustés par le logiciel. 


\subsection{Validité d'un modèle}

II convient d'adjoindre à des critères tels que le coefficient de détermination et son test de signification, des méthodes graphiques, simples d'emploi qui permettent déjà une vérification valable d'un modèle : diagramme de dispersion des couples $\left(X_{i}, Y_{i}\right)$ et graphiques des résidus (TOMASSONE et al, 1983). De plus, il est nécessaire de vérifier par l'utilisation des tests statistiques que les résidus satisfont aux hypothèses d'ajustement d'une régression par la méthode des moindres carrés (BEALL, 1979).

Tableau I : Principaux modeles de rétrocalcul utilisés ( ${ }^{*}$ : in HILE, 1970; ** : in PHILLIPART, 1975) avec $Y=$ longueur du poisson a la capture, $X=$ rayon d'une piece osseuse à la capture, $I=$ áge du poisson, $\nabla_{1}=$ longueur estimée du poisson a l'áge $J$, $X_{1}$ rayon d'une plèce osseuse à l'àge $j, a_{p}, b=$ constantes.

Table I : Principal back-calculating models used (* : In HILE, 1970; ** : in PHILLIPART, 1975) with $Y=$ fish length at the time of capture, $X=$ hard part size at the time of capture, $J=$ fish age $P_{1}=$ estimated fish length at age $J X_{1}=$ hard part size at age $\mathfrak{j}, \mathrm{ap}, \mathrm{b}=$ parameters.

\begin{tabular}{|c|c|}
\hline 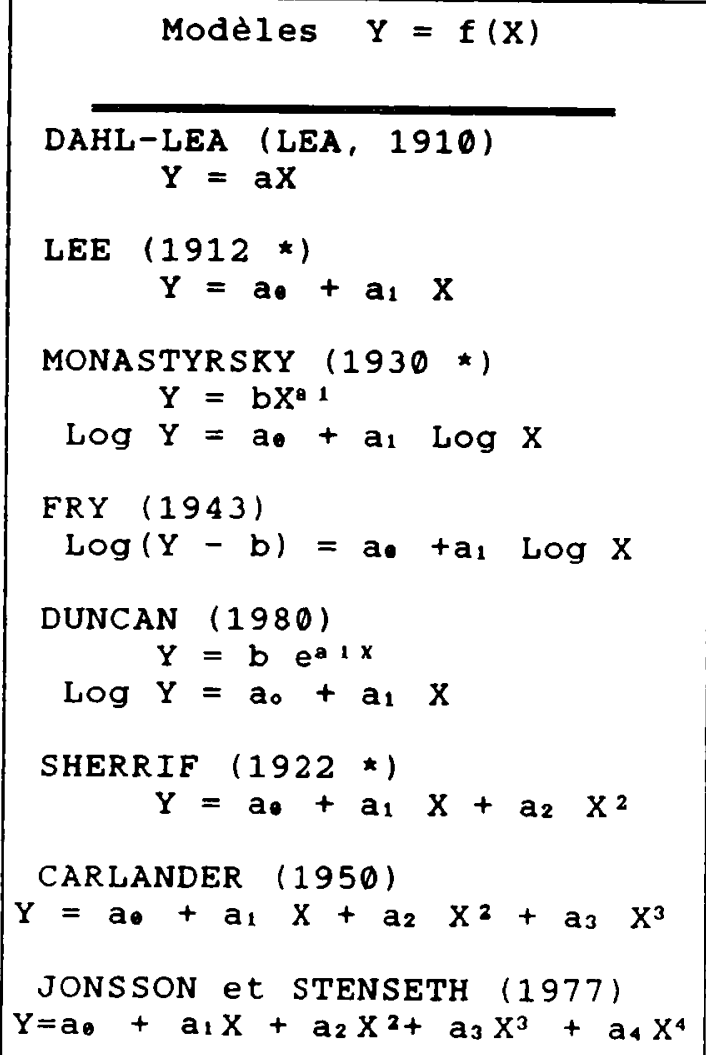 & $\begin{array}{l}\text { FRASER-LEE }(1916,1920 \star *) \\
\hat{\mathrm{Y}}_{1}=\mathrm{a}_{0}+\mathrm{X}_{1} / \mathrm{X}\left(\mathrm{Y}-\mathrm{a}_{0}\right) \\
\text { LECREN }(1947) \\
\operatorname{LOg} \hat{\mathrm{Y}}_{1}=\log Y+a_{1}\left(\log X_{1}-\log X\right)\end{array}$ \\
\hline
\end{tabular}




\subsubsection{Représentation des données}

Avant d'effectuer tout ajustement de modèle sur un jeu de données, la première démarche devrait être d'examiner graphiquement la répartition des points expérimentaux. Cette approche, aussi simple qu'elle puisse paraître, permet déjà d'appréhender la forme du modèle mathématique à choisir, mais aussi de détecter certains problèmes : hétéroscédasticité des résidus (voir paragraphe 1.2.3.), points isolés ou aberrants, sous populations, etc...

La figure $1 \mathrm{~A}$, illustre un cas où a priori l'ajustement d'une régression linéaire n'est pas envisageable. Le cas représenté par la figure 1B, n'exclut pas l'ajustement d'un tel modèle.
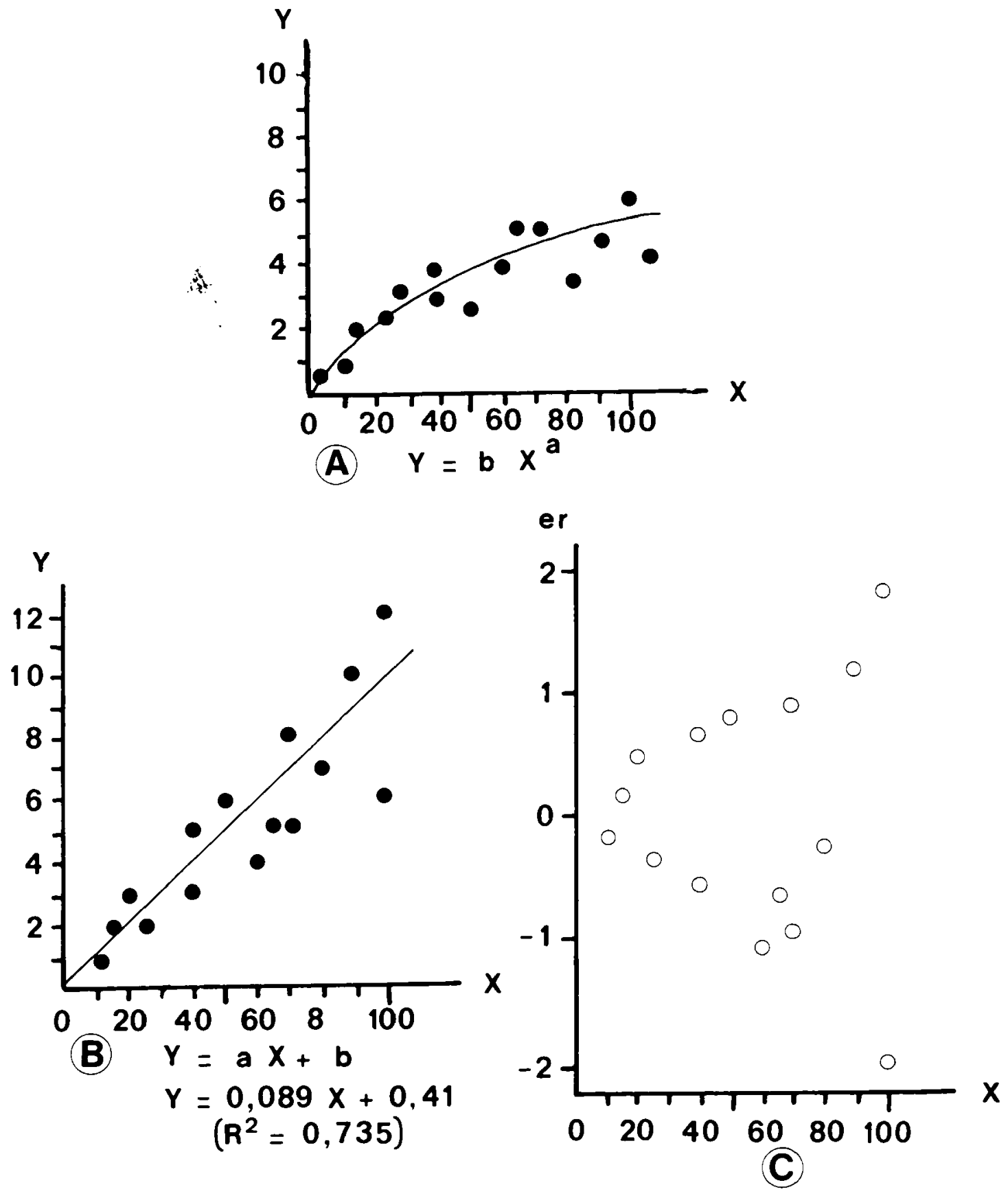

figure 1 : Exemples de diagrammes de dispersion de couples $(X, Y)$ pour des modèles de forme puissance (A) et linéaire (B). Graphe (C) des résidus réduits er en fonction des $X$ pour le modéle linéaire ajusté en $B$.

figure $1:(X, Y)$ scatter plots' examples for curvilinear (A) and linear (B) regressions. Plot (C) of the reduced residuals er against $X$ for the linear model fitted in $B$. 


\subsubsection{Coefficient de détermination}

La variation totale des $Y_{i}$ (longueurs observees des poissons) peut avoir deux origines (SCHERRER, 1984):

- la relation avec $X_{i}$ : une estimation de cette variation, notée SCER, est la somme des carrés des écarts de tous les $\hat{Y}_{i}$ par rapport à la moyenne générale $\bar{Y}$ de tous les $Y_{i}$ observés

$$
\text { SCER }=\sum_{i=1}^{n}\left(\hat{Y}_{i}-\bar{Y}\right)^{2}
$$

- les variations aléatoires des $Y_{i}$ observés pour une même valeur des $X_{i}$. Cette variation appelée résiduelle s'exprime par

$$
\operatorname{SCEE}=\sum_{i=1}^{n}\left(Y_{i}-\hat{Y}_{i}\right)^{2}
$$

La variation totale, notée SCET, est donc la somme SCER + SCEE

$$
\text { SCET }=\sum_{i=1}^{n}\left(Y_{i}-\bar{Y}\right)^{2}
$$

Le coefficient de détermination $\mathrm{R}^{2}$, carré du coefficient de corrélation est défini par

$$
R^{2}=\frac{\text { SCER }}{\text { SCET }}
$$

II exprime la proportion de variation $\hat{Y}_{i}$ expliqué par le modèle. II varie donc entre 0 et 1 . Si la valeur de $\mathrm{R}^{2}$ est voisine de 1 , on dira que $X$ explique bien $Y$ (TOMASSONE et al, 1983).

Pour savoir si cette proportion est significativement différente de 0 pour un risque $\alpha$ on effectue une analyse de variance de la régression. On utilise l'estimation $\hat{F}$ de la variable de FISHERSNEDECOR à $v_{1}=k-1$ et $v_{2}=n-k$ d.d.I.

$$
\begin{aligned}
\hat{F} & =\frac{\text { SCER } /(k-1)}{\text { SCEE } /(n-k)} \\
& =\frac{R^{2} /(k-1)}{\left(1-R^{2}\right) /(n-k)}
\end{aligned}
$$

avec $k=$ nombre de constante du modèle $(k=p+1)$. Si $\hat{F}$ est supérieur au $F_{\alpha}$ (à $v_{1}$ et $V_{2}$ degrés de liberté) de la table, alors le $R^{2}$ est significatif avec un risque d'erreur $\alpha$. Le test de STUDENT, peut être utilisé dans le même but, mais seulement pour les régressions simples (1 variable explicative) (TOMASSONE et al, 1983). C'est pourquoi le choix s'est porté sur le test de FISCHER-SNEDECOR applicable à l'ensemble des modèles proposés par le logiciel (SCHERRER, 1984).

\subsubsection{Analyse des résidus}

S'il existe des tests numériques pour porter un diagnostic sur les résidus, des examens graphiques sont une manière simple et efficace pour mettre en évidence les discordances les plus nettes. On calcule les résidus ei par la formule e $e_{i}=Y_{i}-\widehat{Y}_{i}$ ainsi que les résidus réduits er $i=\left(Y_{i}-Y_{i}\right) / s$ où $\mathrm{s}^{2}$ est la variance résiduelle du modèle $\left(\mathrm{s}^{2}=\mathrm{SCEE} /(n-k)\right)$. On utilise les résidus réduits dans les graphes pour en faciliter l'interprétation.

Les conditions devant être respectées par les résidus sont les suivantes (DRAPER et SMITH, 1966, in BEALL, 1979) : distribution normale, moyenne nulle, variance constante (homoscédasticité) et indépendance. On vérifie ainsi que les hypothèses d'ajustement d'une régression sont satisfaites.

1.2.3.1. Analyse graphique des résidus

Les 3 graphes (JONSSON et STENSETH, 1977 ; TOMASSONE et al, 1983) proposés par le logiciel positionnent les résidus réduits eri en fonction

- des valeurs ajustées $\hat{Y}_{i}$ 
- des valeurs de la variable explicative $X$

- de leur ordre d'acquisition i

Les graphes mettent en évidence les points aberrants qui peuvent être définis commeceux dont le résidu réduit er, n'appartient pas à l'intervalle $(-3 ;+3)$ (ECHEVERRIA, 1987). Les deux premiers graphes permettent de vérifier la normalité et l'homoscédasticité des résidus et le dernier leur indépendance. Ces propriétés des résidus ne peuvent être réalisées que si aucun des 3 graphes ne présente une structure particulière. Par exemple, le graphe présenté à la figure $1 \mathrm{C}$ montre une structure. La variance des résidus n'est pas constante, car elle augmente avec X (hétéroscédasticité). Cela nous oblige à rejeter le modèle linéaire dont le graphe résulte.

On admet (TRANCHEFORT, 1974) qu'avec un risque $\alpha=0,05$, les résidus réduits suivent une distribution normale, de moyenne nulle et de variance constante si dans le cas où $n>30$ :

- $95 \%$ des eriappartiennent à l'intervalle $(-1,96 ;+1,96)$

- $66 \%$ des eriappartiennent à l'intervalle $(-0,95 ;+0,95)$. important.

Ces éléments peuvent être appréciés visuellement sur les graphes sil'échantillon n'est pas trop

Cependant, ces premières observations des graphiques peuventêtre utilement confirmées par une analyse statistique des résidus.

\subsubsection{Analyse statistique des résidus}

Pour tester l'indépendance des résidus, plusieurs tests sont proposésdans la littérature dont le test des suites et celui de DURBIN-WATSON (LABROUSSE 1972). Ce dernier, d'utilisation très simple a été seul retenu. Pour les résidus, classés dans leur ordre d'acquisition i, on calcule la statistique d qui varie entre 0 et 4 :

$$
d=\frac{\sum_{i=2}^{n}\left(e_{1}-e_{i-1}\right)^{2}}{\sum_{i=1}^{n} e_{i}^{2}}=2(1-\rho)
$$

avec $\rho=$ coefficient d'autocorrélation des résidus. Quand d tend vers 0 ou tend vers 4 , on admet qu'il existe une corrélation entre les résidus. Dans le cas où d a une valeur proche de 2 (cela correspondà $\rho \simeq 0$, c'est-à-dire une autocorrélation nulle), on acceptera l'hypothèse d'indépendance (TRANCHEFORT, 1974). La règle de décision pour accepter ou rejeter l'hypothèse d'indépendance fait appel à une table de valeurs critiques (LABROUSSE, 1972).

Les tests de normalité sont nombreux. Cependant celui de KOLMOGOROV-SMIRNOV est cité comme le plus performant et ne nécessite pas de regroupement de classes quand l'effectif de l'échantillon est faible (SIEGEL, 1956 ; SCHERRER, 1984). Le test est basé sur la comparaison de la distribution des fréquences relatives cumulées des résidus $\left(F\left(e_{1}\right)\right)$, classés par ordre croissant, avec celle d'une loi normale théorique $\left(F_{\text {th }}\left(e_{i}\right)\right)$ (SCHERRER, 1984). Soit Dobs $=\max F\left(e_{i}\right)-F_{\text {in }}\left(e_{1}\right)$, pour les probabilités $\alpha$ d'avoir une distribution normale des résidus, les valeurs critiques $D \alpha$ fournies sont (LILLIEFORS, 1967 in SCHERRER, 1984):

$$
D_{0.05}=\frac{0,886}{\sqrt{n}} \quad \text { et } D_{0.01}=\frac{1,031}{\sqrt{n}}
$$

On rejette l'hypothèse de normalité avec le risque avec de première espèce $\alpha$ si Dobs $>D \alpha$.

$S i$ les tests statistiques permettent de vérifier les hypothèses formulées par l'analyse graphique des résidus, ils ne peuvent être utilisés seuls (TRANCHEFORT, 1974). En effet, les tests permettent de rejeter un modèle mais n'apportent aucune indication sur les modifications à apporter (transformation des variables, retrait de données expérimentales aberrantes) pour trouver le meilleur ajustement. Pour détecter les données suspectes, TOMASSONE et ses collaborateurs (1983) propose aussi le calcul des contributions de chaque couple $\left(X_{1}, Y_{i}\right)$. Les points apportant à eux seuls une trop grande contribution à l'ajustement sont suspects même si leur résidu réduit est correct:

$$
(-1,96<\text { eri }<+1,96) \text {. }
$$

On retiendra parmi tous les modèles testés celui ou ceux qui auront satisfait à tous les critè res statistiques et graphiques qui ont été présentés. On admet qu'un modèle est ajusté aux données si le $\mathrm{R}^{2}$ est élevé et significatif, mais surtout si les résidus sont conformes aux hypothèses de normalité et 
d'indépendance, faites lors de l'utilisation d'une régression. Si différents modèles sont validés par tous les critères relatifs aux résidus, on ne choisira pas obligatoirement celui dont le $R^{2}$ est le plus élevé, mais aussi le modèle le plus simple (TRANCHEFORT, 1974). De plus on préfèrera lemodèle ne nécessitant pas de transformation de variables. En effet, dans l'utilisation prédictive d'un modèle établi avec des variables transformées, le retour aux données initiales introduit un biais (TOMASSONE et al, 1983).

\section{UTILISATION D'UN MODÉLE DANS LE RÉTROCALCUL DES TAILLES}

\subsection{Le rétrocalcul des tailles et leur intervalle de prédiction}

Certains ajustements permettent deux méthodes de rétrocalcul des tailles. Par exemple (Tableau I) le modèle linéaire de LEE peut être utilisé directement de façon prédictive ou servir à l'application du modèle de FRASER - LEE. De même le modèle de LECREN trouve son origine dans celui de MONASTYRSKY. Le logiciel propose les différentes méthodes de rétrocalcul pour chaque relation linéarisable $Y=f(X)$ ajustée.

Pour permettre le calcul de l'intervalle de prédiction de chaque taille rétrocalculée $\widehat{Y}_{\mathrm{i}, \mathrm{j}}$ (individu $\mathrm{i}$ à l'âge j) le logiciel fournit l'écart type $\sigma_{i, j}$ associé à cette valeur. Le calcul de $\sigma_{i, j}$ est réalisé pour les différents modèles dans leur forme linéarisée. Pour les régressions de degrés 1 on obtient:

$-\operatorname{pour} \hat{Y}_{i . j}=a_{o}+a_{1} X_{i, j}$ (TRANCHEFORT, $1974 ;$ SCHERRER, 1984)

$$
\sigma_{1, j}=s\left[1+\frac{1}{n}+\frac{\left(x_{i, j}-\bar{x}\right)^{2}}{\Sigma\left(x_{i}-\bar{x}\right)^{2}}\right]^{1 / 2}
$$

$\operatorname{avec} s=[\operatorname{SCEE} /(n-p)]^{1 / 2}$

Pour $\hat{Y}_{i, 1}=a_{0}+\frac{X_{i, j}}{X_{i}}\left(Y_{i}-a_{0}\right)$ on a d'après les propriétés sur les variances:

$$
\sigma_{1.1}=\left[\left[1-\frac{x_{i, j}}{x_{i}}\right]^{2} \cdot v a r \text { ao }\right]^{1 / 2}
$$

avec var ao $=s^{2}\left[\frac{1}{n}+\frac{\bar{x}}{\Sigma\left(x_{i}-\bar{x}\right)^{2}}\right]$

Dans les cas où $Y_{i}$ correspond à une transformation logarithmique de la taille du poisson $i$ (Modèle de MONASTYRSKY), la taille rétrocalculée à l'âge jest estimée par exp $\widehat{Y}_{i, j}$ (TOMASSONE et al., 1983) et son écart type par exp $\sigma i, j$. Il faudra garder à l'esprit que ces nouvelles transformations donnent des estimations biaisées (TOMASSONE et al., 1983) des tailles rétrocalculées et de leur écart type.

Par la suite il est donc possible de déterminer, pour un risque $\alpha$, un intervalle de confiance autour de chaque $\hat{\mathrm{Y}}_{\mathrm{i}, \mathrm{i}}$ :

$$
\left[\hat{Y}_{i, j}-u_{\alpha / 2} \sigma_{i, j} ; \hat{Y}_{i, j}+u_{\alpha / 2} \sigma_{i, j}\right]
$$




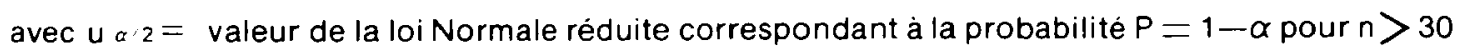

$u_{a: 2}=$ valeur de la loi de Student pour un risque $\alpha$ et $n-p+1$ dd $\sin \leqslant 30$.

\subsection{Domaine de l'application du modèle}

Quand on utilise un modèle prédictif, il est implicitement recommandé que les valeurs prédites $\hat{Y}$ appartiennent à l'intervalle (Ymin; Ymax) des valeurs expérimentales ayant servià ètablir le modèle (Ymin est la plus petite valeur observée et $Y \max$ la plus grande). Cette règle peut souffrir des exceptions mais alors, cela implique des risques d'erreur plus importants. Pour le cas présent, ECHEVARRIA (1987) suggère d'appliquer cette règle. En tout état de cause, il est admis que l'échantillon sur lequel est établi le modèle doit comporter des individus des âges pour lesquels les tailles vont être rétrocalculées (CARLANDER, 1985). RICKER (1973) suggère, de plus, que chaque classe d'âge doit avoir, un poids équivalent dans l'échantillon. En effet, il a été mis en évidence. notamment chez la Truite (SMITH, 1955 ; KIPLING, 1962) que la relation $Y=f(X)$ était différente pour les premiers stades de croissance du poisson. Le logiciel signale donc les tailles rétrocalculée $\hat{Y}$ qui sortent de la gamme des tailles observées (Ymin ; Y max)

\subsection{Tailles moyennes par àge et par classe d'àge}

Les tailles étant rétrocalculèes pour chaque poisson i à chaque âge j, le logiciel calcule les longueurs moyennes des poissons, $\vec{Y}_{1}$, par âge j et par classe d'âge. Les tailles moyennes sont accompagnées de leur variance, représentative de la variabilité inter-poisson, mais n'incluant pas la variabilité due au modèle.

Le biologiste n'a pas toujours la possibilité d'effectuer des rètromesuressur les écailles de tous les individus composant son échantillon. On trouve donc dans le logiciel une option qui permet d'extrapoler les résultats obtenus sur un sous-échantillon à l'échantillon total. Pour utiliser cette option, il faut simplement fournir la décomposition en classes de tailles, voire mieux en classes de tailles par classe d'âge, de l'échantillon total. Le logiciel nécessite que le sous-échantillon possède au moins un individu de chaque groupe de la partition de l'échantillon. Un programme utilitaire, mis au point pour exécuter cette décomposition, permet à l'utilisateur de choisir l'intervalle de classe de tailles à sa convenance. Le modèle $Y=f(X)$ est ajusté sur le sous échantillon. Les tailles rètrocalculées pour ce dernier se verront affecter un poids, fonction de leur degré de représentativité de l'échantillon total. Les tailles moyennes parâge et par classe d'âge et leurvariance sont donc estimées par pondération des tailles rétrocalculées connues.

\section{EXEMPLE : RÉTROCALCUL DES TAILLES POUR UN ÉCHANTILLON DE JUVÉNILES DE TRUITE DE MER}

\subsection{Matériel et méthodes}

Lors de la période de dévalaison de 1987 (28/02 au 29/05) 239 juvéniles de Truite (Salmo trutta L.) ont été capturès par piégeage sur le Douet de la Taille, affluent de la Touques, rivière de Basse Normandie. La plupart des individus, âgés de 1 an à $2^{-}$ans, (Tableau II) étaient essentiellement des smolts et présmolts de Truite de mer.

Pour chaque individu, la longueur fourche $Y$ a été mesurée en $\mathrm{mm}$ et des écailles ont été prélevées sur la zone standard définie pour le saumon atlantique (BAGLINIËRE, 1985), c'est-à-dire dans les 6 premiers rangs au-dessus de la ligne latérale, et autour d'une ligne joignant le bord postérieur de la nageoire dorsale au bord antérieur de la nageoire anale. Pour chaque poisson, 3 écailles (KERR, 1961) exemptes de toute régénération ont fait l'objet de mensurations (grossissement 50 - projecteur de film Projectina muni d'un micromètre) sur le plus grand rayon de la partie antérieure de l'écaille, exprimées en gradation de micromètre (500 gradations $=1 \mathrm{~cm})$.

$\mathrm{X}=$ rayon au moment de la capture

$\mathrm{X}_{1}=$ rayon au premier annulus

$\mathrm{x}_{2}=$ rayon au second annulus

Pour l'ajustement d'un modèle $Y=f(X)$ et les rétrocalculs de tailles, les mensurations moyennes (KERR, 1961 ; HILE, 1970) pour 3 écailles ont été utilisées afin de s'affranchir en partie de la variabilité intra - site des écailles.

Afin de tester les "différentes options" du logiciel nous avons extrait un sous-échantillon de 155 individus (Tableau II) de l'échantillon total. 
Tableau II : Répartition en classes de taille et par cohorte de l'échantillon de 239 juvénilles migrants de Truite de mer et du sous-échantillon de 155 individus.

Table II : Length-frequency distribution for each cohort of 239 young migrating sea Trout Sample and of the sub-sample of 155 fishes.

\begin{tabular}{|c|c|c|c|c|c|c|c|c|}
\hline \multirow{4}{*}{\multicolumn{2}{|c|}{$\underset{(\mathrm{mm})}{\text { Classes de taille }}$}} & \multicolumn{7}{|c|}{ Effectifs } \\
\hline & & \multicolumn{3}{|c|}{ Echantillon total } & \multicolumn{4}{|c|}{ Sous-échantilion } \\
\hline & & 1 et $1+$ an & 2 et $2+$ ans & Total & & et $1+a n$ & $\mid 2$ et $2+a n s \mid$ & Total \\
\hline & & $----\cdots----$ & 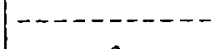 & $-\cdots$ & & $---\cdots---$ & --------- & $-\cdots$ \\
\hline 120 & -129 & 2 & 0 & 2 & & 2 & 0 & 2 \\
\hline $130-$ & -139 & 1 & 0 & 2 & & 1 & 0 & 1 \\
\hline 140 & -149 & 2 & 1 & 3 & & 2 & 1 & 3 \\
\hline $150-$ & -159 & 11 & 2 & 13 & & 8 & 2 & 10 \\
\hline $160-$ & -169 & 8 & 9 & 17 & & 7 & 8 & 15 \\
\hline $170-$ & -179 & 2 & 18 & 20 & & 2 & 11 & 13 \\
\hline $180-$ & -189 & 2 & 42 & 44 & & 1 & 25 & 26 \\
\hline $190-$ & -199 & 2 & 48 & 50 & & 2 & 32 & 34 \\
\hline $200-$ & -209 & 0 & 43 & 43 & & 0 & 21 & 21 \\
\hline $210-$ & -219 & 0 & 21 & 21 & & 0 & 14 & 14 \\
\hline $220-$ & -229 & 0 & 15 & 15 & & 0 & 8 & 8 \\
\hline $230-$ & -239 & 0 & 7 & 7 & & 0 & 5 & 5 \\
\hline $240-$ & -249 & 0 & 1 & 1 & & 0 & 1 & 1 \\
\hline $250-$ & -259 & 0 & 2 & 2 & & 0 & 2 & 2 \\
\hline Tota & al & 30 & 209 & 239 & & 25 & 130 & 155 \\
\hline
\end{tabular}

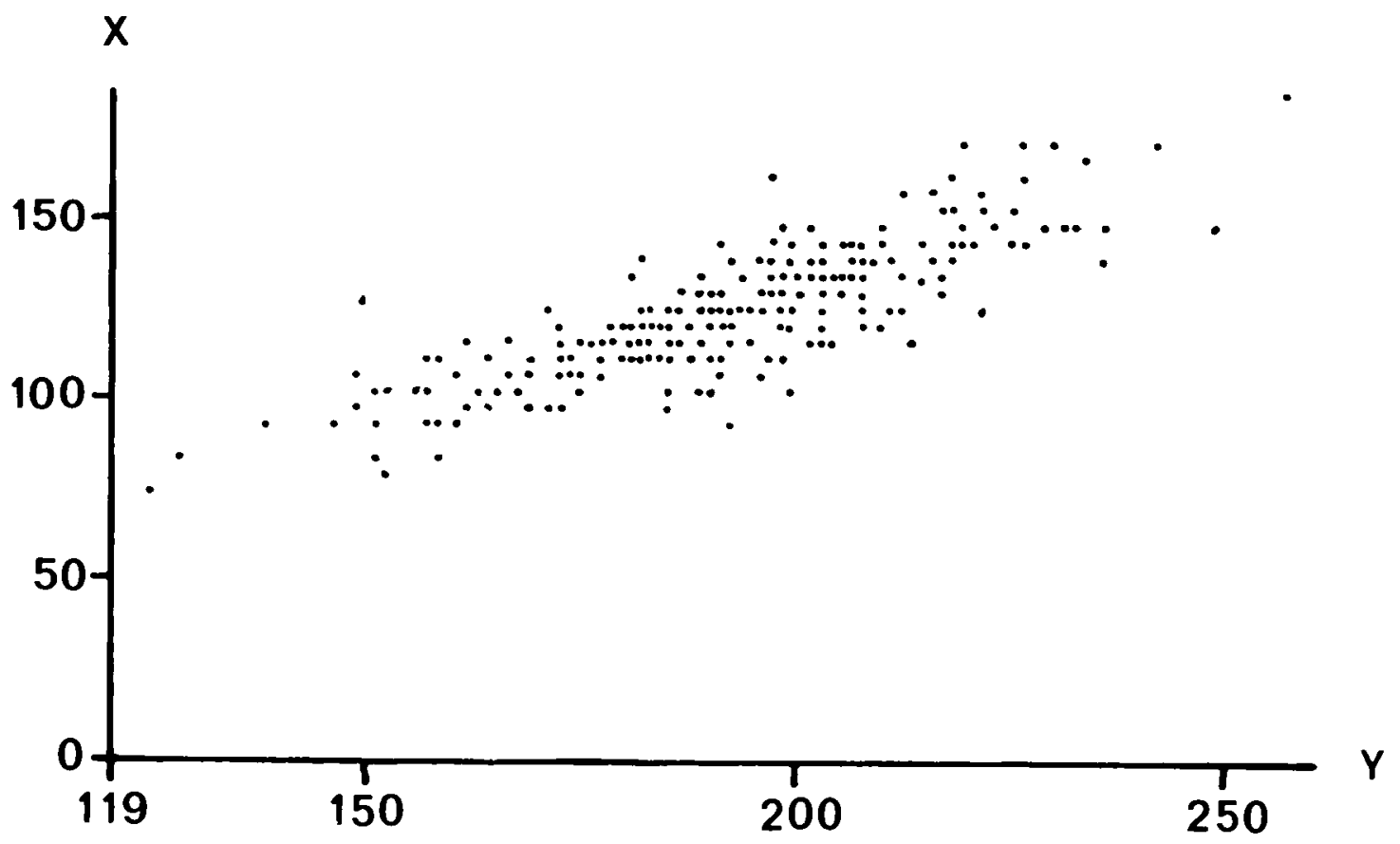

figure 2 : Diagramme de dispersion des couples $\left(Y_{1}, X_{i}\right)$ pour un échantillon de 239 juvéniles migrants de Truite de mer. Avec $Y_{1}$ : longueur fourche du poisson (mm) et $X_{i}$ : rayon moyen de la partie antérieure des écailles (unité arbitraire).

figure $2:\left(X_{1}, Y_{i}\right)$ scatter plot for a 239 young migrating sea Trout. With $Y_{1}:$ fish fork length $(\mathrm{mm})$ and $X_{i}$ : mean radius of anterior part of scales (arbitrary unit). 
3.2. Choix de la meilleure relation longueur du poisson - rayon de l'écaille, $Y=f(X)$

Le positionnement des points $\left(Y_{1}, X_{i}\right)$ dans un plan (fig. 2) nous permet de supposer que le meilleur ajustement sera vraisemblablement une régression linéaire. Cependant parmi tous les modèles testés, 5 peuvent dans un premier temps retenir notre attention car ils présentent des coefficients de détermination équivalents et hautement significatifs pour le test F de FISCHERSNEDECOR :
$Y=67,46+1,0062 X$
$\mathrm{R}^{2}=0,712$
$\log Y=2,078+0,660 \log X$
$\mathbf{R}^{2}=0,718$
$\log Y=4,591+0,0053 X$
$\mathrm{R}^{2}=0,702$
$Y=20,013+1,776 X-0,0031 X^{2}$
$\mathrm{R}^{2}=0,717$
$Y=-45,83+3,370 X-0,016 X^{2}+0,00003 X^{3}$
$\mathrm{R}^{2}=0,701$

\subsubsection{Examen graphique des résidus}

Pour chaque ajustement (sauf celui du modèle exponentiel), l'examen des 3 graphes des résidus (paragraphe 1.2.3.1.) ne fait apparaître aucune structure particulière ; ils ont tous une allure similaire. A titre d'exemple, nous présentons à la figure 3, les graphes des résidus réduits erı en fonction des $\mathrm{Xi}$ observés et celui en fonction de leur ordre d'acquisition i pour la régression linéaire.
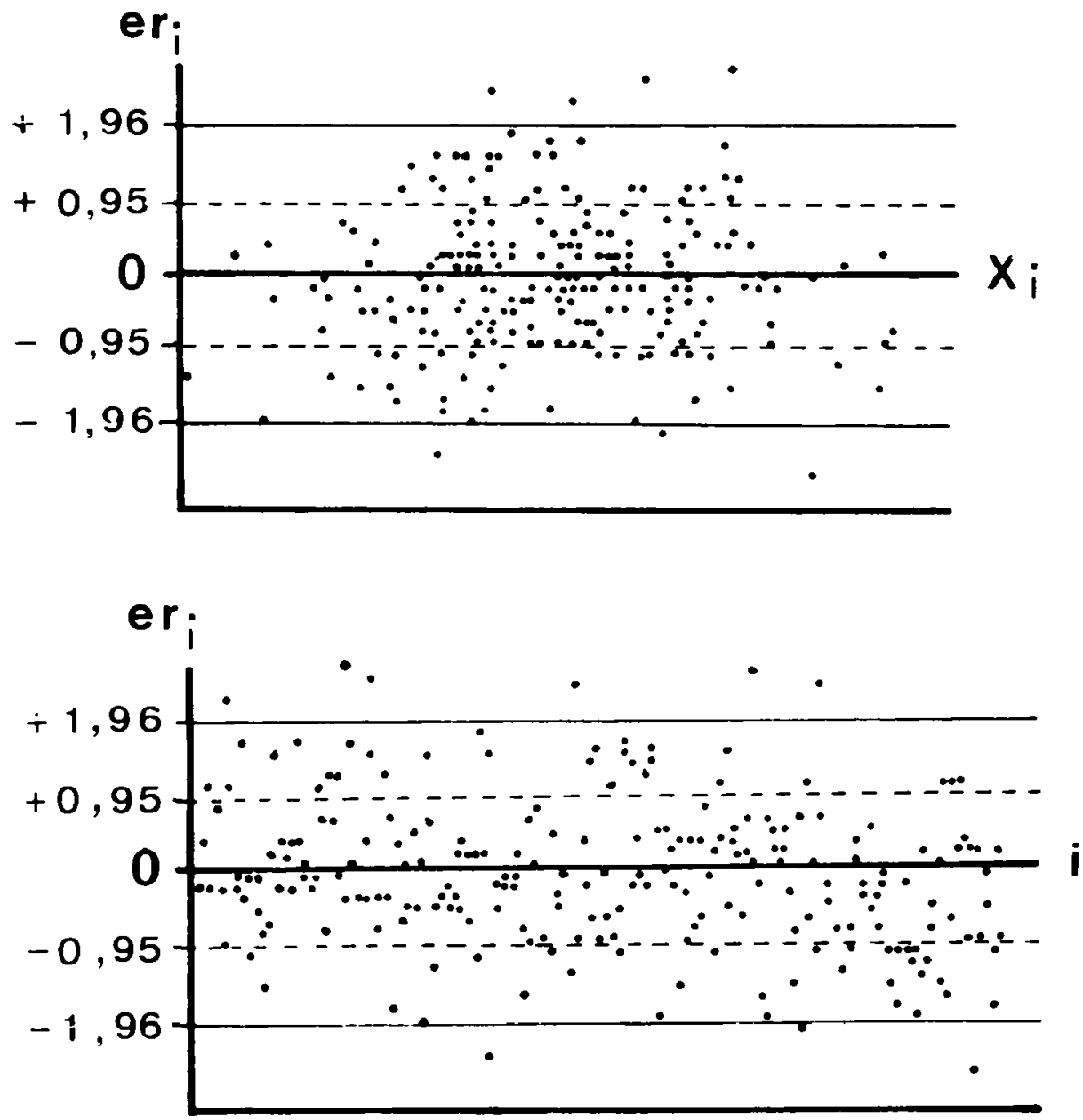

figure $3 \quad$ : Graphiques des résidus réduits (eri) en fonction des rayons moyens des écailles $\left(X_{i}\right)$ et en fonction de leur ordre d'acquisition $i$ pour le modèle ajusté $Y=67,46+1,0062 X$ sur l'échantillon total de 239 juvéniles migrants de Truite de mer.

figure 3 : Reduced residuals (eri) plotted against mean scale radii $\left(X_{i}\right)$ and against time sequence $i$ for the model $Y=67,46+1,0062 X$ fitted on the 239 individuals total sample of 239 young migrating sea trout. 
Le tableau III, où sont présentés pour chaque ajustement, les pourcentages de er appartenant aux intervalles $(-0,95 ;+0,95)$ et $(-1,96 ;-1,96)$ met en évidence que 3 modèles satisfont aux conditions de normalité des résidus exposées au paragraphe 1.2.3.1. Ce sont les modèles linaire et polynomiaux d'ordre 2 et 3.

Tableau III : Critères statistiques permettant de choisir la meilleure des 4 relations ajustées entre la longueur fourche du poisson $Y$ et le rayon de l'écaille $X$ pour 239 juvénilles de Truite de mer.

Table III : Statistical criteria to choose the best of the 4 relations fitted between fish folk length $Y$ and scale radius $X$ for 239 young sea trout.

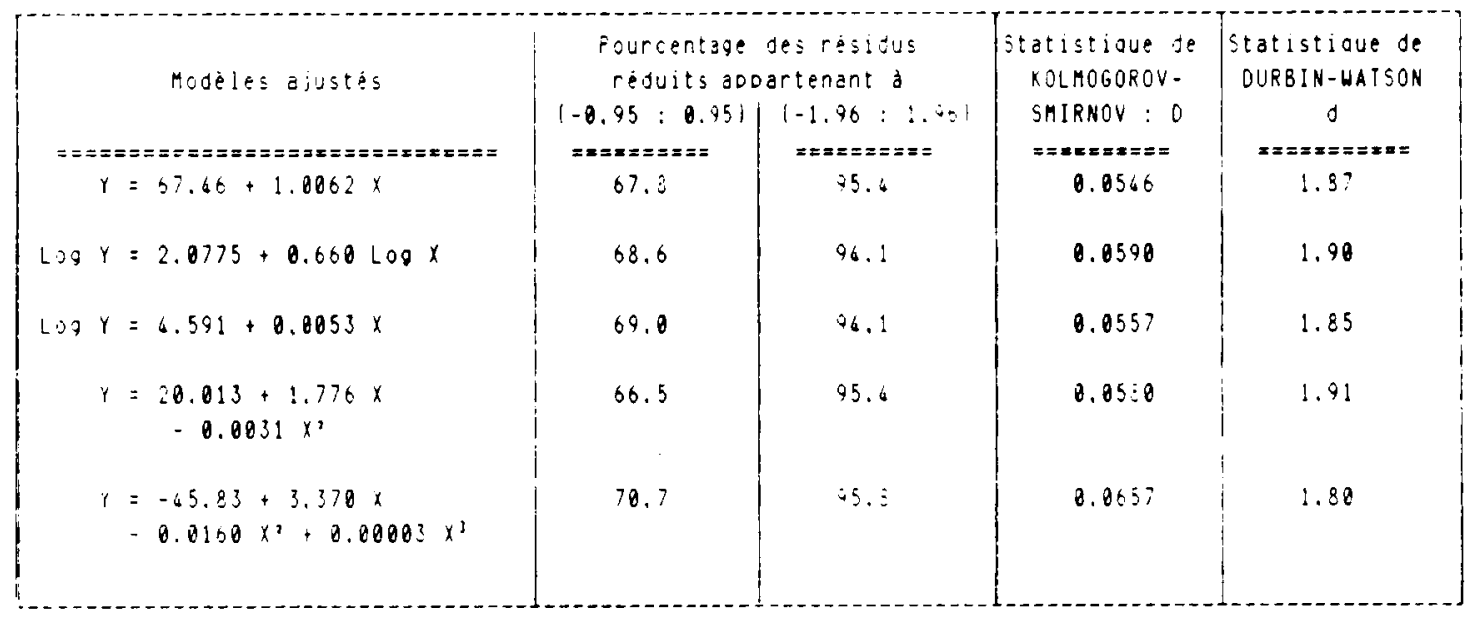

\subsubsection{Analyse statistique des résidus}

Pour les trois modèles pouvant encore être retenus, le logiciel fournit les statistiques d de DURBIN-WATSON (test d'indépendance) et D de KOLMOGOROV-SMIRNOV (test de normalité) (Tableau III). Seul le modèle linaire satisfaitaux conditions requises pour les statistiques $d(1,69<d<$ 2,31 ) et $D(D<0,057)$, pour un risque $\alpha=0,05$.

La meilleure relation entre la longueur fourche du poisson $Y$ et le rayon antérieur de l'écaille $X$ est donc, pour l'échantillon étudié une régression linaire:

$$
Y=67,46+1,0062 x
$$

On vérifie, qu'aucun point n'est isolé $(-3<$ eri $<+3$, quel que soit $i)$ et n'apporte une contribution particulière à la régression.

\subsection{Rétrocalcul des tailles}

Pour le modèle ajusté et dont la validité a été démontrée, deux méthodes de rétrocalcul des tailles $Y_{i, j}$ à l'âge $j$ sont possibles pour chaque individu $i$ :

méthode de LEE

$\hat{Y}_{i . j}=67,46+1,0062 X_{i, j}$ méthode de FRASER-LEE

$\hat{Y}_{i, j}=\frac{X_{i . j}}{X_{1}}\left(Y_{1}-67,46\right)+67,46$

Les tailles rétrocalculées à 1 an, pour quelques poissons d'âges différents à la capture, reflètent les résultats obtenus pour la totalité de l'échantillon (Tableau IV).

On constate que par la méthode de LEE, les écarts-types sont élevés (précision de la prédiction de l'ordre de $15 \%$ pour $\alpha=0,05)$ mais du même ordre de grandeur pour tous les individus. Cette dernière caractéristique est une conséquence directe de l'utilisation prédictive d'une régression linéaire. En effet la région de confiance d'une droite est délimjtée par deux branches d'hyperbole (TOMASSONE et al., 1983). L'écart type minimum autour de $\hat{Y}_{i, j}$ est obtenu pour $X_{i . j}=\bar{X}$ et il s'accroît d'autant plus que la différence entre $X_{i, j}$ et $\bar{X}$ est importante (CARLANDER, 1981). Dans les exemples du Tableau IV, les valeurs des $X_{i, 1}$ étant proches, celles des 
Tableau IV : Longueurs rétrocalculées à 1 an $\left(\widehat{\gamma}_{1}\right)$ et leur écart type $\sigma 1$ associé, pour 4 individus et 2 modèles. Résultats pour les régressions obtenues sur l'échantillon total (A) et sur le sous-échantillon (B).

Table IV : Back-calculated length at 1 year $\left(\hat{Y}_{1}\right)$ and their standart deviation $\left(\sigma \sigma_{1}\right)$ for 4 fishes and 2 models. Results are given for the models obtained with the total samples (A) and with the sub-sample (B).

\begin{tabular}{|c|c|c|c|c|c|c|}
\hline & \multicolumn{2}{|c|}{$\begin{array}{c}\text { Caractéristiques } \\
\text { la capture }\end{array}$} & \multicolumn{2}{|c|}{$\begin{array}{l}\text { Méthode de LEE } \\
\hat{Y}_{?}=a_{2}+a_{1} X:\end{array}$} & \multicolumn{2}{|c|}{ 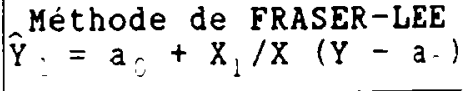 } \\
\hline & $\begin{array}{c}\text { Longueur } Y \\
(\mathrm{~mm})\end{array}$ & Age & $\hat{Y}:(m m)$ & $\therefore:(\mathrm{mm})$ & $\hat{Y}_{1}(m m)$ & $\sigma_{1} \quad(\mathrm{~mm})$ \\
\hline$A$ & $\begin{array}{l}128 \\
172 \\
218 \\
259\end{array}$ & $\begin{array}{l}1 \\
1+ \\
2 \\
2+\end{array}$ & $\begin{array}{l}151 \\
165 \\
136 \\
156\end{array}$ & $\begin{array}{l}12,01 \\
11,94 \\
12.12 \\
11,98\end{array}$ & $\begin{array}{l}128 \\
157 \\
135 \\
159\end{array}$ & $\begin{array}{l}0.00 \\
0.74 \\
2.87 \\
2.74\end{array}$ \\
\hline B & $\begin{array}{l}128 \\
172 \\
218 \\
259\end{array}$ & $\begin{array}{l}1 \\
1+ \\
2 \\
2+\end{array}$ & $\begin{array}{l}146 \\
162 \\
130 \\
152\end{array}$ & $\begin{array}{l}12,30 \\
12,20 \\
12,46 \\
12,26\end{array}$ & $\begin{array}{l}128 \\
155 \\
127 \\
151\end{array}$ & $\begin{array}{l}0.00 \\
0.91 \\
3.55 \\
3,39\end{array}$ \\
\hline
\end{tabular}

écarts types associés aux $\widehat{Y}_{i, 1}$ le sont aussi. La région de confiance de la droite ajustée sur l'échantillon étudié (fig. 4A) montre que l'intervalle de confiance est équivalent dans la gamme des $\hat{Y}$ observés.

Par la méthode de FRASER - LEE, les écarts-types sont nettement moins élevés (la précision la plus mauvaise est de l'ordre de $5 \%$ pour $\alpha=0,05$ ). Cela s'explique par l'élimination de la variabilité due à la constante $a_{1}\left(a_{1}=1,0062\right)$ dans le calcul des écarts-types associés aux valeurs prédites. Ces derniers s'accroissent avec la différence entre $X_{i, \text {, et }} \bar{X}$ (fig. 4B).

De plus, pour un individu âgé de 1 an au moment de sa capture (exemple $Y=128 \mathrm{~mm}$ du Tableau IV), le modèle prédictif de LEE donne $\widehat{Y}_{1}=Y_{1}$ alors que par celui de FRASER - LEE on obtient $\widehat{Y}_{1} \neq Y_{1}$. Cette dernière méthode de rétrocalcul permet en outre de s'affranchir de la variabilité de la taille des écailles pour des poissons de même longueur et repose sur le principe que, la croissance de l'écaille est proportionnelle à celle du corps (CARLANDER, 1985).

Ces différentes considérations, nous font préférer la méthode de FRASER - LEE pour le rétrocalcul des tailles. De plus les tailles rétrocalculées à $1 \mathrm{an}$, les plus petites $\left(Y_{i .1} \mathrm{~min}=91\right)$ sont proches de la gamme des $Y_{i}$ observés $\left(Y_{1} \min =119 ; Y_{1} \max =259\right)$. Nous pouvons donc utiliser pour des études écologiques, les tailles moyennes par âge et par classe d'àge calculées par le logiciel (Tableau V).

\subsection{Utilisation de l'option "sous-échantillon"}

La même démarche que précédemment nous conduit à retenir le modèle linaire qui satisfait à tous les critères. La relation longueur du poisson $(Y)$ - rayon de l'écaille $(X)$ obtenue pour les 155 individus du sous-échantillon est la suivante:

$$
Y=53,30+1,1209 x \quad R^{2}=0,751
$$

Quelle que soit la méthode de rétrocalcul utilisée, les tailles rétrocalculées à 1 an sont statistiquement identiques (Tableau IV) à celles estimées à partir de l'échantillon total. Les intervalles de confiance autour des valeurs prédites sont du même ordre de grandeur.

Enfin, les tailles moyennes par âge et par classes d'âge, calculées par pondération des résultats obtenus sur le sous-échantillon, sont équivalentes à celles obtenues par l'analyse de l'échantillon total (Tableau V). 


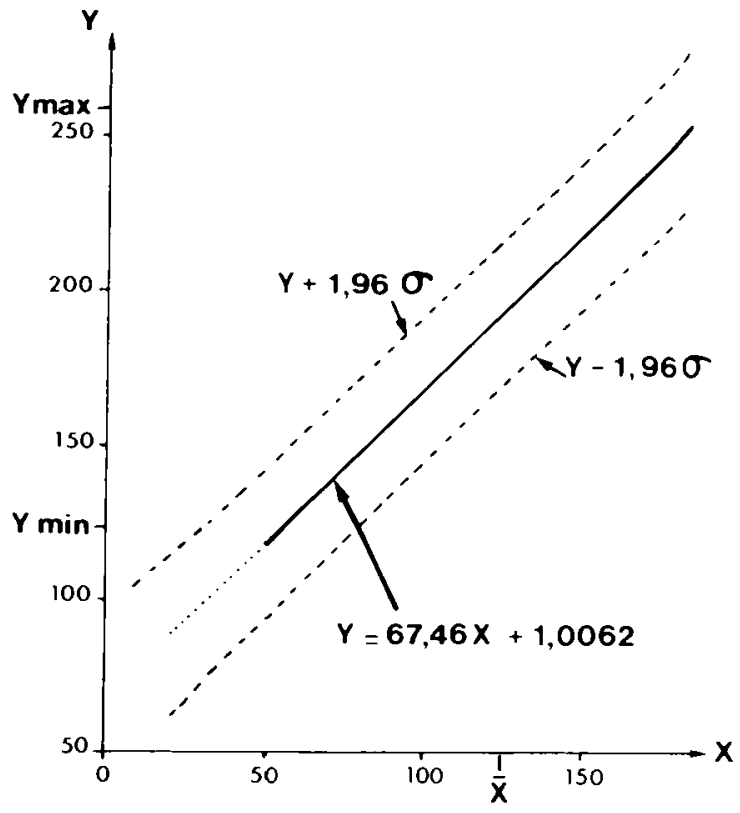

A : LEE

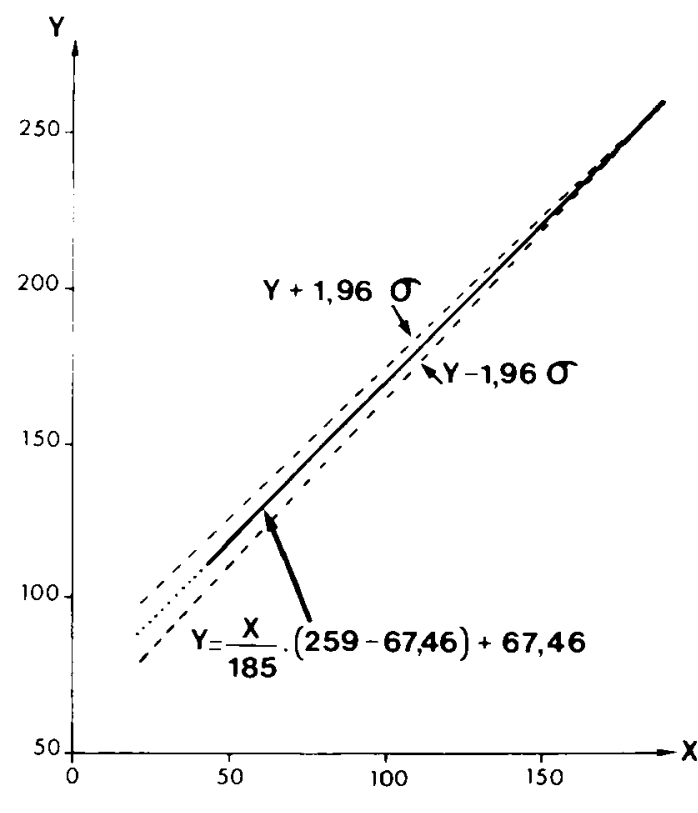

B : FRASER - LEE

figure 4 : Région de confiance des modèles de LEE (A) et FRASER-LEE (B) pour l'échantillon total de 239 juvénilles migrants de Truite de mer. Avec $\bar{X}=$ moyenne des $X$ observés et $Y \min , Y \max =$ Longueurs minimale et maximale observées.

figure 4 : Confidence région of LEE (A) et FRASER-LEE (B) models for the total sample of 239 young migrating sea trout. $\bar{X}=$ mean of $X$ observed and $Y \min , Y \max =$ minimal and maximal lengths observed.

Tableau $\mathbf{V}$ : Taille moyenne rétrocalculée $\hat{Y}_{j}$ et écart type $\sigma ;$ par âge $\mathbf{j}$ et par classe d'âge obtenus, par le modèle de FRASER-LEE, avec l'échantillon total de juvénilles migrants de Truite de mer (A) et avec un sous-échantillon de 155 individus après pondération des résultats $(B)$.

Table $V \quad$ : Mean back-calculating length $\hat{\gamma}_{j}$ and standart de deviation, $\sigma ;$ for each age $j$ and each age class, obtained by FRASER-LEE model, with young migrating sea Trout total sample (A) and with the sub-sample of 155 fishes after weighting of the results $(B)$.

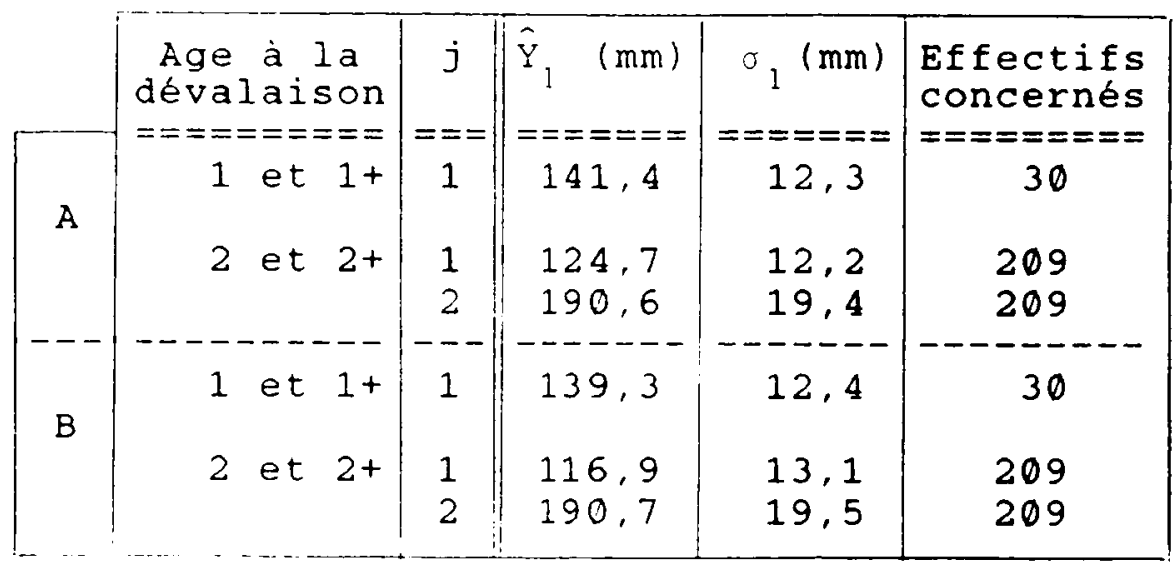




\section{CONCLUSION - DISCUSSION}

Même si nous ne l'avons pas évoqué précédemment, il est évident que la validité d'un modèle dépend de la "qualité" des données qui ont servi à l'établir. Certains principes sont acceptés avant d'ajuster un modèle de rétrocalcul:

- si les pièces anatomiques utilisées sont des écailles, elles doivent toutes être prélevées sur un même site, défini avec précision (KIPLING, 1962 ; HILE, 1970) et mesurées de la même façon (CARLANDER, 1982). Même avec ces précautions, on n'est pas à l'abri de difficultés pour ajuster un modèle compte tenu de la variabilité intra-site des écailles (HILE, 1970).

- l'ajustement d'une régression suppose que les variances des résidus soient constantes (conditionnellement aux estimations), ce qui est rarement le cas dans le domaine qui nous intéresse. En effet la variance de la longueur du poisson augmente quand le rayon de la pièce anatomique s'accroit (MOTTLEY, 1942 in WHITNEY et CARLANDER, 1956). C'est pourquoi on peut trouver des avantages à ajuster des modèles curvilinéaires pour lesquels une transformation logarithmique des variables permet de stabiliser les variances de ces dernières (TOMASSONE et al., 1983). Cependant WHITNEY et CARLANDER (1956) estiment que, pour les objectifs poursuivis par la plupart des études, la précision obtenue avec une régression linaire simple. est suffisante pour se dispenser de la transformation logarithmique des variables, et admettre que les variances des longueurs des poissons sont les mêmes pour chaque valeur du rayon de l'écaille.

- CARLANDER (1981), met l'accent sur la nécessitè d'établir un modèle sur un échantillon "correct" et "bien distribué" c'est-à-dire contenant des petits poissons dont la taille est inférieure ou égale à la plus petite taille rétrocalculèe à 1 an et des grands poissons. Ces considérations sur la constitution de l'échantillon rejoignent nos propros relatifs au champ d'application d'un modèle de rétrocalcul "prédictif". Pour la Truite de mer par exemple, SYCH (1967) a montré qu'il était nécessaire d'utiliser deux modèles de rétrocalcul (un pour la phase juvénile et un pour la phase marine) car les modalités de croissance des écailles évoluent dans le temps.

Le logiciel que nous avons élaboré, n'étant qu'un outil de calcul, ne "porte aucun jugement" sur la qualité des données. II signale cependant certaines utilisations "abusives" du modèle (longueurs rétrocalculées en dehors de la gamme des longueurs observées). L'ensemble des tests statistiques proposés n'est pas exhaustif. Nous avons retenu, parmi les plus souvent utilisés, ceux qui sont considérés comme les plus robustes par les statisticiens.

Outre toutes les méthodes de rétrocalcul nécessitant l'ajustement d'un modèle $Y=f(X)$, le logiciel permet l'utilisation du modèle isométrique de DAHL - LEA et celui de FRASER - LEE avec une constante ao "standard". De plus dans l'estimation des tailles moyennes par âge et par classe d'âge, le programme fait ces calculs par sexe, si ils sont connus. Il peut être intéressant de comparer les 2 courbes de croissance élaborées à partir des tailles rétrocalculées.

Ce logiciel est composé de 4 programmes en langage FORTRAN 77 et nécessite des fichiers en code ASCII. Le rétrocalcul des tailles et les calculs statistiques sont réalisés par 2 programmes. Deux utilitaires effectuent respectivement le positionnement des couples $(X, Y)$ du fichier de données et la décomposition en classe de taille et d'âge de l'échantillon. Afin que les programmes exécutables occupent moins de $360 \mathrm{~K}$ octets de mémoire, ce qui permet d'utiliser plus facilement le logiciel sur tout ordinateur compatible PC ne possédant que deux lecteurs de disquettes, l'échantillon ne pourra être supérieur à 1.000 individus âgés de 20 ans au maximum.

\section{BIBLIOGRAPHIE}

BAGLINIERE, J.L., 1985. La détermination de l'âge par scalimétrie chez le Saumon Atlantique Salmo salar dans son aire de répartition méridionale : utilisation pratique et difficultés de la méthode. Bull. Fr. Pêche Piscic., 298, 69-105.

BEALL E., 1979. Analyse scalimétrique d'une population de Truite de mer. Salmo trutta L., des lles Kerguelen, TAAF : structure d'àge, croissance, reproduction. Thèse Doc. Biol. Anim., (Hydrobiologie), Fac. Sci. Toulouse, $182 \mathrm{p}$.

CARLANDER K.D., 1950. Growth rate studies of Saugers, Stizostedion canadense canadense (Smith) and yellow perch. Perca flavescens (Mitchill) from lake of the woods, Minnesota, Trans. Am. Fish. Soc., 79, 30-42.

CARLANDER K.D., 1981. Caution on the use of the regression method of back-calculating lengths from scale measurements, Fisheries, 6 (1), 2-4.

CARLANDER K.D., 1982. Standart Intercepts for calculating lengths from scale. Measurements for some Centrarchid and Percid fishes, Trans. Am. Fish. Soc., 111, 332-336.

CARLANDER K.D., 1985. Sampling problems in deriving a body scale regression for growth calculation from fish scale, Verh. Internat. Verein. Limnol., 22, 2534-2536. 
DUNCAN K.W., 1980. On the back-calculation of fish lengths; modifications and extensions to FRASER - LEE equation, J.Fish Biol., 16, 725-730.

ECHEVERRIA T.W., 1987. Relationship of otolith length to total length in rockfishes from northern and central California, Fish. Bull., 85 (2), 383-387.

FRIE R.V., 1982. Measurement of fish scales and back-calculation of body lengths using a digitizing pad and microcomputer, Fisheries, 7 (6), 5-8

FRY F.E.J., 1943. A method for the calculation of the growth of fishes from scale measurements, Publ. Ontario Fish. Res. Laboratory, 61, 5-18.

HAUT H., 1982. Mathèmatiques et statistiques - Programmes en basic, Ed du PSI, Lagny-sur-Maine (France), $272 \mathrm{p}$.

HILE R., 1970. Body scale relation and calculation of growth in fishes, Trans. Amer. Fish. Soc., 3 , 468-474.

JONSSON B. and STENSETH N.S., 1977. A method for estimating fish length from otolith size, Report Instit. Freshw. Res. of Drottningholm, 56, 81-86.

KERR R.B., 1961. Scale to length ratio, age and growth of Atlantic Salmon in Miramichi fisheries. J. Fish. Res. Bd. Canada, 18 (1), 117-124.

KIPLING C., 1962. The use of scales of the trout (Salmo trutta L.) for the back-calculation of growth, J. Cons. Int. Explor. Mer, XXVII, 3, 304-315.

LABROUSSE C., 1972. Introduction à l'économétrie, Ed. DUNOD, Paris, 150 p.

LEA E., 1910. On the methods used in the Herring investigation. Publ. de circ. - CIEM, 53, 7-25.

LEBART L., MORINEAU A. et FENELON J.P., 1982. Traitement des données statistiques - méthodes et programmes. Ed. DUNOD, Paris, $510 p$.

LE CREN E.D., 1947. The determination of the age and growth of the perch (Perca fluviatilis) from the opercular bone. J. Anim. Ecol., 16, 188-204.

PHILLIPPART J.C., 1975. Dynamique des populations de poissons d'eau douce non exploitées, 291-394. In LAMOTTE M. et BOURLIËRE F. : problèmes d'écologie : la démographie des populations de vertébrés, Masson et Cie Ed., Paris, 443 p.

RICKER W.E., 1973. Linear Regressions in Fishery Research, J. Fish. Res. Bd Can., 30 (3), 409-434.

SCHERRER B., 1984. Biostatistiques, Ed. Gaëtan Morin, Chicoutimi (Québec), 850 p.

SIEGEL S., 1956. Nonparametric statistics for the behavorial sciences. Ed. MacGraw-Hill book Company. New York, $312 p$.

SMITH S.B., 1955. The relation between scale diameter and body length of kamloops trout, Salmo gairdneri kamloops. J. Fish. Res. Bd Canada, 12 (5).

SYCH R., 1967. Dependance of scale radius and body weight or length of sea trout (Salmo trutta L.) from Vistula river. Roczn. Nauk. Roln., H90 (2), 327-339.

TOMASSONE R., LESQUOY, E. et MILLIER C., 1983. La régression, nouveaux regards sur une ancienne méthode statistique, INRA actualités scientifiques et agronomiques, 13, Ed. Masson, Paris, $177 \mathrm{p}$.

TRANCHEFORT J., 1974. La régression - application à l'agronomie, Bureau d'études statistiques de I'ITCF, Ed. ITCF, Paris, $178 \mathrm{p}$.

WITHNEY R.R. and CARLANDER K.D., 1956. Interprétation of body-scale regression for computing body length of fish. J. of Wildlife Manag., 20 (1), 21-27. 


\title{
DISCOURS DE CLOTURE
}

\author{
(A. FOSTIER)
}

Exercice difficile, pour quelqu'un qui n'est pas un écologiste, que de conclure sur un Colloque aussi riche. Riche de par la diversité des thèmes abordés, ou bien évoqués dans les discussions et qui n'ont pu être développés faute de temps. Riche aussi de par la diversité des origines géographiques et professionnelles des participants. Nous avons pu juger de travaux réalisés au Canada, en Suisse, en Belgique, au Portugal et dans de nombreux territoires français, y compris les îles Kerguelen. Sont par ailleurs représentés ici : le Conseil Supérieur de la Pêche, le Centre d'Etude du Machinisme Agricole, du Génie Rural et des Eaux et Forêts, le Centre National de la Recherche Scientifique, des Universités, des Ecoles Nationales Supérieures Agronomiques et I'Institut National de la Recherche Agronomique. Ce colloque répondait à un réel besoin d'information et de confrontation d'idées, en permettant de réunir des chercheurs, des conseillers en gestion piscicole et quelques gestionnaires, ces derniers devant être les utilisateurs des travaux présentés.

Vous serez, je pense, d'accord pour conclure avec moi que ce Colloque sur la Truite est une réussite. Il doit, de par les échanges qu'il a provoqués, permettre d'améliorer la qualité des données collectées et la pertinence du choix des variables observées. Je vous renvoie, à ce sujet, au débat sur les critères et les modèles de croissance. Il devrait également permettre d'améliorer les outils de collecte de ces données et en assurer une meilleure exploitation par les chercheurs et les gestionnaires. Ce n'était pas seulement un point, nécessaire, sur une question, mais l'occasion d'ouvrir ou d'élargir de nouvelles réflexions. Je citerai, comme exemple, les discussions animées sur la prise en compte nécessaire, dans les travaux à venir, de l'écologie des espèces congénères et de l'habitat dans un sens très large.

Il est également apparu, au cours de ces trois journées, qu'un effort devrait être fait pour que les recherches futures s'enrichissent davantage d'acquis venant d'autres disciplines que l'écologie. Nous l'avons vu avec les exposés d'éthologistes, de généticiens des populations, ou encore l'analyse historique de Max THIBAULT. Les débats ont montré, par ailleurs, l'intérêt qu'il y aurait à collaborer pius étroitement avec des statisticiens, des pathologistes, des nutritionnistes, des physiologistes.

Un autre effort devrait porter sur la clarification des objectifs. Au vu de tel ou tel travail, on se pose parfois la question de savoir s'il s'agissait de décrire, d'expliquer, de prédire ou de gérer. La définition et le choix pertinent des objectifs, avant d'agir, sont indispensables pour ces travaux parfois longs et coûteux en moyens humains et matériels.

Je terminerai en exprimant l'espoir que ce colloque ne sera pas sans prolongement; qu'il suscitera de nouvelles coopérations, de nouveaux échanges d'informations et d'idées, afin de vous permettre de mieux chercher, de mieux conseiller, de mieux gérer.

\section{A. FOSTIER}

Directeur de la Station de Physiologie et d'Ecologie des Poissons

(Institut National de la Recherche Agronomique) 


\section{ANNEXES}




\title{
OUVRAGE PARU - NEW BOOK
}

Nous avons le plaisir de vous annoncer la parution d'un ouvrage de synthèse complétant les numéros 318 et 319 du B.F.P.P. et intitulé :

\author{
"Biologie et écologie de la truite (Salmo trutta L.) en France" \\ J.L. BAGLINIERE et G. MAISSE éditeurs. \\ Collection Hydrobiologie et Aquaculture de l'I.N.R.A.
}

\section{Table des matières*}

Introduction : La Truite commune (Salmo trutta $L$ ) : son origine, son aire de répartition, ses intérêts économique et scientifique

J.L. BAGLINIERE.

\section{1'e Partie : La Truite de rivière}

1.1. Biologie de la Truite commune (Salmo trutta L.) dans les rivières françaises G. MAISSE, J.L. BAGLINIERE

1.2. L'habitat de la Truite commune (Salmo trutta L.) en cours d'eau J. HAURY, Dominique OMBREDANE et J.L. BAGLINIERE

1.3. Stratégie alimentaire de la Truite commune (Salmo trutta L.) en eaux courantes A. NEVEU

1.4. Organisation sociale et territorialité chez la Truite commune immature au cours de l'ontogénèse M. HELAND

\section{$2^{\mathrm{e}}$ partie : Plasticité écologique et diversité génétique chez la Truite}

2.1. Principales caractéristiques de la biologie de la Truite (Salmo trutta L.) dans le Léman et quelques affluents

A. CHAMPIGNEULLE, B. BUTTIKER, P. DURAND et M. MELHAOUI

2.2. La Truite de mer (Salmo trutta L.) en Normandie-Picardie

G. EUZENAT, Françoise FOURNEL et A. RICHARD

2.3. Diversité génétique et gestion des populations naturelles de truite commune R. GUYOMARD

\section{$3^{\text {e }}$ partie : La Gestion des populations naturelles de Truite}

3.1. La gestion des populations naturelles de truite commune en France analysée dans une perspective historique (1669-1986)

M. THIBAULT

Conclusion : Connaître les bases biologiques de la gestion, une idée toujours d'actuaiité pour la truite (Salmo trutta L.)

G. MAISSE, J.L. BAGLINIERE

* Pour la bonne information de chacun, nous publions les résumés des articles de l'ouvrage annoncé.

Vente: I.N.R.A. Service des publications Route de Saint Cyr - 78000 VERSAILLES. 


\section{INTRODUCTION : LA TRUITE COMMUNE (SALMO TRUTTA L.), SON ORIGINE, SON AIRE DE REPARTITION, SES INTÉRÊTS ÉCONOMIQUE ET SCIENTIFIQUE}

\section{J.L. BAGLINIERE}

Station de Physiologie et d'Ecologie des Poissons, I.N.R.A., 65, route de Saint Brieuc - 35042 RENNES Cedex

\section{RESUME}

La Truite commune répartie dans le monde sous différentes formes et dans divers milieux (rivière, lac, estuaire et mer) est une seule espèce Salmo trutta $\mathrm{L}$. à caractère migrateur facultatif. Originaire du continent européen, elle a été introduite avec succès dans au moins 24 pays. La valeur économique de cette ressource nationale et internationale repose sur l'engouement de sa pêche sportive, l'importance de sa pêche professionnelle et son intérêt pour l'aquaculture marine.

Actuellement le cycle biologique de la Truite commune et les paramètres s'y rattachant sont relativement bien connus. Par contre, sa dynamique de population et sa stratégie démographique, au travers des interactions entre les différentes formes et de l'impact des activités humaines, restent difficiles à appréhender. Ainsi, avant la mise en place de telles études, il est apparu nécessaire de faire un bilan des connaissances acquises sur la biologie de la truite en France.

\section{SUMMARY}

The Brown Trout present in the world in different forms and environments (river, lake, estuary, sea) is only one species with a optional migratory character. Originally native of the european continent, it has been successfully introduced in at least twenty-four contries. The economical value of its national and international resource is related to the infatuation of its rod fishing, to the importance of its commercial fishing and to its interest in sea farming.

Now, the life history of Brown Trout and the related parameters are enough well known. In contrary, its population dynamics and demographic strategy in relation to the interactions between the different forms and to the influence of human activities, lack yet for informations. So, before the settlement of such studies, it was necessary to make the point of the knowledges obtained on the Biology of the Brown Trout in France. 


\title{
BIOLOGIE DE LA TRUITE COMMUNE (SALMO TRUTTA L.) DANS LES RIVIËRES FRANÇAISES
}

\author{
G. MAISSE et J.L. BAGLINIËRE
}

Station de Physiologie et d'Ecologie des Poissons, I.N.R.A.,

65, route de Saint Brieuc - 35042 RENNES Cedex

\section{RÉSUME}

La présente communication fait le point sur les études ayant été menées sur la truite commune du bassin du Scorff, de 1973 à 1984. Les principaux résultats obtenus sont les suivants:

- la croissance individuelle varie suivant le milieu (ruisseau - rivière; amont - aval) et l'année; les différences s'établissent très précocement et l'effet année est particulièrement lié à la température,

- chez les géniteurs le rapport des sexes est toujours en faveur des mâles (en majorité âgés de 1+ an); chez les femelles, la grande majorité mature pour la première fois en $2_{+}$, cependant dans les ruisseaux, les femelles $1+$ sont fréquentes. La maturation en $1+$ dépend de la croissance en $0_{+}$.

- le frai a lieu dans les affluents pour la partie aval du bassin et à la fois dans la rivière principale et les affluents dans la partie amont,

- les taux de survie ont pu être évalués sur un ruisseau; ils confirment que la grande majorité des mortalités est enregistrée en $0_{+}$; chez les géniteurs la survie annuelle des femelles est meilleure $(50 \%)$ que chez les mâles $(30 \%)$.

Un cycle biologique est proposé, tenant compte de l'existence d'une génération intermédiaire dans les affluents, venant se placer entre les géniteurs migrants de la rivière principale et les juvéniles $(1+$ et $2+$ ) produits par les ruisseaux et assurant l'essentiel du recrutement en truites dans le Scorff.

Au niveau national, les travaux sont essentiellement ponctuels; ils vont dans le sens des observations réalisées sur le Scorff, et permettent de classer les régions en trois catégories de croissance (forte, moyenne et faible).

Une réflexion sur les perspectives de recherche privilégie deux thèmes :

- la "capacité biogènique" du milieu, selon LEGER (1910)

- les stratégies démographiques.

\section{SUMMARY}

This communication takes stock of studies carried out on the common trout in the Scorff basin from 1973 to 1984 . The principle results are the following:

- individual growth depends on the environment (stream-river; upstream-downstream) and the year. The differences are established very early on and the "year effect" is particulary linked to temperature,

- in mature adults there is always proportionally more males (the majority aged $1+$ years). In females the large majority mature for the first time at the 2+ stage, however, in the streams $1+$ females are frequent. Maturation at $1+$ depends on growth at $0_{+}$.

- Spawning take place in tributaries in the downstream part of the basin and either in the main part of the river or its tributaries for the upstream part,

- survival rates were evaluated in a stream, confirming that the large majority of deaths were recorded at $0_{+}$. In the mature fish the annual survival rates of females is better $(50 \%)$ than for males $(30 \%)$.

A biological cycle is suggested, taking into account the existance of an intermediate generation in the tributaries, which are placed between the mature migrating fish in the principle river and the juvenile fish $(1+$ and $2+)$ produced in the streams and ensuring the essential recruitment of trout in the Scortf.

On the national level, results are similar to those obtained in the Scorff, thus enabling a classification of regions into three categories of growth (large, medium and small).

Two themes come to light through this research:

- the "biogenic capacity" of the environment, according to LEGER (1910)

- demographic strategies. 


\title{
L'HABITAT DE LA TRUITE COMMUNE (SALMO TRUTTA L.) EN COURS D'EAU
}

\author{
J. HAURY, Dominique OMBREDANE et J.L. BAGLINIÉRE \\ Station de Physiologie et d'Ecologie des Poissons, I.N.R.A., \\ 65 , route de Saint Brieuc - 35042 RENNES Cedex
}

\section{RÉSUME}

L'analyse de l'habitat de la truite commune (Sa/mo trutta L.) en rivière correspond à plusieurs niveaux d'étude depuis l'aire de répartition jusqu'au microhabitat. Cette espèce est rhéophile, exigeante pour la qualité d'eau et l'oxygénation: elle est une sténotherme d'eau froide.

Des différentes zonations longitudinales des cours d'eau proposées dans la littérature, il ressort que la zone trutticole est caractérisée par une pente importante et une température estivale inférieure à $20-22^{\circ} \mathrm{C}$. Elle est constituée de secteurs de largeur et d'ordre de drainage très variables. A l'intérieur de cette zone, les densités de truite commune et notamment des $0_{+}$augmentent de l'aval vers l'amont, ce qui peut être mis en parallèle avec la localisation des zones de frai dans les têtes de bassins.

A l'échelle des macrohabitats, les frayères sont localisées en zones peu profondes, à courant assez important et sur des substrats meubles mais grossiers; leurs dimensions et la granulométrie sont proportionnelles à la taille des géniteurs, les individus les plus gros frayant plus en aval et en milieu plus profond. Pour les stades libres, on constate que les juvéniles se trouvent principalement dans les zones courantes peu profondes (radiers et rapides), alors que les adultes occupent des sites plus profonds, à vitesse de courant moindre et granulométrie plus hétérogène. La structuration du territoire de la truite en microhabitats complémentaires, correspondant à des fonctions particulières (abri, repos, alimentation,...), est une manifestation de la compétition intraspécifique, notamment pour les juvéniles. La compétition interspécifique est également un élément influençant la population de truites. Compte tenu de l'inféodation relative de la truite à certains types d'habitats, des modèles prédictifs du stock en fonction des caractéristiques du milieu ont pu être établis.

Les variations naturelles cycliques ou accidentelles des caractéristiques de l'habitat déterminent des déplacements de population et peuvent modifier l'équilibre des cohortes, exprimant ainsi une grande variabilité des relations habitat-poisson. Les impacts sur l'habitat trutticole des altèrations physiques du lit et des berges, des modifications de débit et des aménagements piscicoles sont précisés : toute diminution de l'hétérogénéité du milieu physique a généralement des répercussions négatives sur la population de truite.

Une homogénéisation des méthodes et protocoles d'étude est une nécessité pour une meilleure mise en évidence des relations entre la truite et son milieu de vie. Certains axes de recherche sont proposés pour mieux quantifier la capacité d'accueil des rivières et gérer les populations via l'habitat.

\section{SUMMARY}

The understanding of the habitat of the common trout (Salmo trutta L.) in rivers involves several levels of study, from the distribution area up to the microhabitat. The common trout is a rheophile species, demanding high water quality and oxygenation, and a stenotherm of cold water.

It is apparent from the literature about longitudinal zonation of streams that trout populate segments characterised by a large gradient and a summer temperature of less than $20-22^{\circ} \mathrm{C}$. These have very variable widths and drainage orders. Inside this zone, common trout densities, notably the $0_{+}$individuals, increase in the direction of downstream to upstream, which parallels the localisation of spawning zones occuring at the heads of the basins.

On the macrohabitat level, the redds are localised in relatively shallow areas, with quite strong currents and on course substrates. The dimensions of redd and granulometry of the substrates are proportional to the size of the mature fish, larger individuals reproducing further downstream and in deeper waters. For the free stages, juveniles are found mainly in shallow zones with strong currents (riffles and rapids), whereas adults occupy deeper sites, with weaker currents and more heterogeneous granulometry. The partition in various microhabitats of trout territory corresponds to particular functions (shelter, rest, food, etc.) and is a demonstration of intraspecific competition, particularly in juvenile trout. Interspecific competition is an element which also equally influences the trout population. Prediction models of stock can be established, with function to the characterisics of the habitat, taking into account the relative attachment of trout to certain types of habitat. 
Natural or accidental cyclic variations in the habitat wich determine movement of the population and which may modify the equilibrium of the cohorts, demonstrate a large variability in the habitat-fish relationship. The impact on the trout habitat of physical alterations in the river bed and bank, modifications in water flow and factors due to fish farming are identified: all decreases in the heterogeneity of the physical medium has, generally, negative repercussions on the trout population.

Consistency of experimental methods and protocols is necessary for a better understanding of the relationship between the trout and its environment. Certain research themes are suggested for better quantifying the capacity of rivers and using the habitat in order to manage populations. 


\title{
STRATEGIE ALIMENTAIRE DE LA TRUITE COMMUNE (SALMO TRUTTA L.) EN EAUX COURANTES
}

\author{
A. NEVEU \\ Station de Physiologie et d'Écologie des Poissons, I.N.R.A., \\ 65 , route de St Brieuc - 35042 RENNES Cedex
}

\begin{abstract}
RÉSUME
L'étude des apports énergétiques est primordiale pour la compréhension du fonctionnement d'un écosystème salmonicole. Les sources de nourriture pour la truite sont représentées surtout par les invertébrés benthiques et aériens dont l'estimation des stocks reste complexe de par leur variabilité spatio temporelle.

L'analyse des contenus stomacaux, associée à des mesures de laboratoire sur le transit stomacal, permet une estimation de la consommation journalière dans la nature à condition d'effectuer des prélèvements réguliers dans le temps en respectant les rythmes atimentaires. De même, l'âge des truites a une influence non seulement sur les quantités ingérées, mais aussi sur leur qualité.

Des mesures calorimétriques plus précises et plutôt expérimentales permettent une évaluation des dépenses énergétiques et les pertes par excrétion pour une approche des bilans énergétiques applicable aux conditions naturelles.

En fait, les méthodes actuelles d'investigation restent trop imprécises quant à l'évaluation correcte de la consommation des poissons sauvages comparativement aux estimations de la productivité benthique.
\end{abstract}

\section{SUMMARY}

Study of fish population trophic dynamics is necessary to know the influence of various parameters on production in wiew of a better human harwest. But the understanding of fish production must be replaced within the knowledge of a whole ecosystem approach. The difficulties of measuring many parameters can explain the scarcity of trophic studies on salmonid streams.

Trout is carnivore with a majority of invertebrates in the dict and sometimes small fishes. In streams the principal origin of food items is the benthic invertebrates fauna completed by exogenous terrestrial casualities in relation to the diversity of banks flora. Consumption of terrestrial insects increases with the trout age.

In spite of numerous studies it is very difficult to calculate the invertebrates secundary production and the part accessible to the trout. Spatial heterogenity of benthic populations, temporal variability of density, sampling methods, are unable to allow an exhaustive approach.

Daily food catches of trout may be estimated by field data on stomach contents. In attempting to abtain good results it is important to have measurements applicable to whole population, with a range of size classes, times and seasons.

Actual results show that food selection is not a hazardous process. Size, mobility, density, morphological aspect of preys, but also capacity of learning of the trout interact to a foraging optimized. On the other hand the consumption intensity is subordinated to the stomach repletion and in nature it is difficult to know if maximum ration can be often obtained.

In aquatic environment many factors are periodical and the life of many organisms is dependent on these oscillations. So the invertebrates drift (often nocturnal) have a daily rhythm which makes access to numerous preys standing in hiding places during the light period. Many studies show that the trout is foraging on drift, but results in rivers of Pyrenees's mountains show a foraging activity directly on benthic preys. As a matter of fact the feeding rhythm of trouts is correlated to temperature which regulate the number of daily meals and if the temperature is adequate foraging is more or less continuous.

Knowledge of feeding rhythms associated to stomacal evacuation rates may approach the daily consumption. Models of stomacal evacuation are calculated from experimental ways in laboratory and extrapolated to field conditions. So these methods applied to a trout river in Pyrenees's mountains give a daily consumption between 195 and $6269 \mathrm{cal} / \mathrm{Trout} /$ day in june, in other words between 0,4 and 6,4 percent of individual medium weights of each age and in october between 177 and $1086 \mathrm{cal} /$ trout/day or 0,1 to 1,2 percent of medium weights. 
Energy budgets in the field requires more energy and more data which can only be obtained in the laboratory with many methodological problems. In the absence of direct informations it is generaly necessary to apply experimental models. So in a Pyrenees's river the energy disponibilities for growth is between 0,8 and 1,7 percent of individual medium weights in june but only between 0.2 and -0.4 percent in october, season with an effective decrease in stoutness. In all cases the extrapolation of laboratories data in the wild may be executed with many cautions.

In data of some studies the trout consumes numerous times the standing crop of invertebrates ("Allen's paradox"). Moreover the impact of fish predation is not obvious on diversity or density of the benthos. As a matter of fact these results show the inadequacy of actual methods to estimate not only the secundary productivity of benthic invertebrates but also the intensity of fish foraging. And so the complexity of all energy studies can explain the rarity of data in natural conditions. 


\title{
ORGANISATION SOCIALE ET TERRITORIALITÉ CHEZ LA TRUITE COMMUNE IMMATURE AU COURS DE L'ONTOGÉNÉSE
}

\author{
M. HELAND \\ Laboratoire d'Ecologie des Poissons, Station d'Hydrobiologie, \\ I.N.R.A., B.P. 3, 64310 SAINT-PEE-SUR-NIVELLE
}

\begin{abstract}
RÉSUMÉ
A partir d'une synthèse des résultats obtenus en milieu naturel ou dans des ruisseaux artificiels en conditions contrôlées, le processus de mise en place de l'organisation sociale est présentée depuis l'émergence en relation avec les ressources du milieu. Cette présentation s'appuie sur l'analyse des différentes phases de développement des relations sociales chez l'alevin de truite au sortir de la frayère qui vont conduire à l'établissement d'une première mosaïque territoriale. L'évolution de la mosaïque territoriale au cours de l'ontogénèse vers un système plus souple d'utilisation des ressources spatiales et alimentaires préfigure les relations hiérarchiques observées chez les adultes. Le rôle de la structure sociale, territoriale et/ou hiérarchique, est défini par rapport à l'ajustement de la densité de population aux ressources du milieu, ainsi que par rapport aux déplacements des juvéniles ou la dévalaison précoce des alevins.
\end{abstract}

\section{SOCIAL ORGANIZATION AND TERRITORIALITY OF IMMATURE BROWN TROUT DURING ONTOGENY}

\section{SUMMARY}

Social and territorial behaviour of fry and immature juveniles were analysed mostly under controlled conditions in artificial streams presenting the morphodynamic characteristics of Salmonid spawning grounds. Some observations in the natural environment were made in small brooks of the Nivelle watershed.

The study of territorial settlement in an artificial stream showed that, after emergence and the learning of static swimming behaviour, the fry oriented themselves near the bottom not only with respect to the water current in relation to the substratum but also with respect to the available food represented mainly by invertebrate drift. Competition occurred for the best site in relation to water current conveying the food and a social hierarchy was established. According to the definition of Wickler (1976), site attachment occurred thanks in part to food reinforcement. From this site, called station, the fry attacked all intruders. Some twelve days after emergence, a mosaïc of exclusive territories was also created in relation to the substratum with a preference for heterogeneous areas. In such a mosaïc, dominant fry were territorial tenants and subordinate fry were obliged to hide under stones or to move out.

In artificial streams, a two-fold increase of the input of natural drifting food did not result in an increase of the number of territories. In another experiment, the increase in initial fry density did not increase the number of territories in the stream. In the salmonid stream ecosystem, with a particular substratum, a well defined maximum number of territories was established at a given stage of the fish development, even under ad libitum food conditions. The social and territorial behaviour of Salmonid juveniles seemed to be a behavioural adaptation to the trophic use of the environment particularly with respect to drifting invertebrates. It is an energy-based model of territoriality.

Downstream moving fry observed very soon after emergence played an important regulating role during the adjustment of the territorial mosaïc to the trophic conditions : indeed when food was unlimited, the number of territories stayed at the same level, but subordinates were more numerous. Inversely, where the food was scarce, an important downstream movement occurred and, consequently, a lower number of marginal subordinates was observed in the stream. In controlled conditions, precocious downstream migrants ultimately adopted the static swimning behaviour and settled within the territorial mosaic, but they did it later and were smaller than non-migrants.

During ontogeny, the territorial mosaic of young fry a few weeks after emergence was replaced by a system of trophic and habitat uses of space and time called partial territories. Dominance relationships held on but subordinates occupied small marginal territories. This social organization was probably modified during the night and seemed to be given up during winter. These results are discussed in relation to behavioural plasticity in brown trout exhibited very early during ontogeny. 


\title{
PRINCIPALES CARACTÉRISTIQUES DE LA BIOLOGIE DE LA TRUITE (Salmo Trutta L.) DANS LE LÉMAN ET QUELQUES AFFLUENTS
}

\author{
A. Champigneulle (1), B. Buttiken (2), P. DuRANd (3) et M. MELHAOUi (4)
}

(1) I.N.R.A., Station d'Hydrobiologie Lacustre, B.P. 511, 75 Avenue de Corzent, 74203 THONON (France)

(2) Conservation de la Faune, Chemin du Marquisat 1, CH 1025, ST SULPICE (Suisse)

(3) ECOTEC, rue Listard 5, 1202 GENEVE (Suisse)

(4) Université Mohammed $1^{\text {er }}$, Faculté des Sciences, Département de Biologie - OUJDA (Maroc)

\begin{abstract}
RÉSUMÉ
L'article présente les principales caractéristiques de la biologie de la truite de lac dans le Léman, grand lac franco-suisse de 58.240 ha en cours d'eutrophisation. La croissance en lac, très nettement supérieure à celle en rivière, est forte et semble en liaison avec un régime ichtyophage. L'examen des écailles montre l'existence de 1 ou 2 années de croissance initiale faible de type rivière. La reproduction des truites de lac a généralement lieu de la fin novembre à la fin janvier. principalement dans les affluents du lac; elle apparait légèrement plus tardive que celle des truites résidentes. Les géniteurs migrants à reproductions multiples restent généralement attachés au même cours d'eau. II y a davantage de jeunes géniteurs (2-3 ans) chez les mâles que chez les femelles et il existe des truites immatures des deux sexes âgées de 3 ans et plus. De récentes études indiquent que des déversements en affluents d'alevins prégrossis peuvent contribuer à la production de truites de lac dont certaines reviennent comme géniteurs à la rivière de relâcher.
\end{abstract}

\section{MAIN CHARACTERISTICS OF THE BIOLOGY OF THE BROWN TROUT (SALMO TRUTTA L.) IN LAKE LEMAN AND SOME TRIBUTARIES}

\section{SUMMARY}

The biology of brown trout (Salmo trutta L.) has been studied in Lake Leman, a large (58240 ha) mesoeutrophic French-Swiss lake, and in the downstream part of some medium-sized (width 3 to $10 \mathrm{~m}$ ) spawning tributaries. The yearly total catches of lake dwelling trout, mainly realized in the lake, varied between 12 and $38 \mathrm{t}$. Several hundred thousands of yolk sac and, mainly, prefed fry from wild (lake dwelling spawners) or domestic origin are released each year in the tributaries or right in the Lake.

The autumnal resident trout population present in the studied tributaries is mainly composed of $0_{-}$and $1+$ juveniles at the following densities: 11 to $720+/ 100 \mathrm{~m}^{2}$ and 2 to $211+/ 100 \mathrm{~m}^{2}$. In the places with prefed fry restocking (13 to $50 \mathrm{ind} / 100 \mathrm{~m}^{2}$ ) a large proportion (28 to $93 \%$ of 0 - and 10 to $91 \%$ of $1+$ ) are issued from stocking. The growth of juveniles in tributaries is good as the mean total length is $83-117 \mathrm{~mm}$ for $0_{+}$and $164-209 \mathrm{~mm}$ for 1 . Some downstream movements to the lake occured during the "autumn-winter-beginning of spring" period and they concerned mainly juveniles with one or two growing seasons.

The study of scales from lake dwelling trouts indicates the presence of one (type 1) or two (type 2) (occasionaly 3 : type 3 ) initial years with a slower "river type" growth. A, the same age, the mean length of type 1 lake dwelling trouts is significantly larger than the one of type 2 trouts. The difference exists from early stages as the back calculaded mean length at one year is bigger (129 $\mathrm{mm}$ ) for type 1 than for type $2(100 \mathrm{~mm})$ trouts. The growth is much more better for lake than for river dwelling trouts. The important growth in the lake is partly linked to the fact that trouts eat fish juveniles (roach mainly perch) in the lake.

For resident trouts the first maturity can occured at 2 years ( 1 - stage) for both sexes but it is only at 3 years $(2+$ stage) that almost all resident trouts are mature. In section of tributaries opened to sport-fishing the sex-ratio is in favour of males and there are very few spawners of 3 years or more. In closed sections there are much more old spawners and the sex-ratio is equilibrated. The first maturity is generally obtained later for lake dwelling trout and immatures of both sexes aged of 3 or 4 years are frequently found in pelagic catches of lake dwelling trouts. The relative fecundity of females varies between 1300 and $3700 \mathrm{ova} / \mathrm{kg}$ of female and is $2460 \mathrm{ova} / \mathrm{kg}$ in average. The age 
structure of lake dwelling trout spawners has been studied during five consecutive years in the river Redon, a small (length : $10 \mathrm{~km}$; width : 4-5 m) tributary on the French shore. The age of lake dwelling trout spawners varied between 2 and 7 years. Among males, there was a noticeable (41\%) but fluctuating (15 to $72 \%$ according the year) percentage of young spawners of 2 years old. The percentage of spawners of 3 years was similar (27-31\%) for both sexes. The percentage of old (4 to 7 seven years) spawners was much more important for females $(67 \%)$ than for males $(27 \%)$. The percentage of spawners of type 1,2 or 3 was respectively 68,31 and $1 \%$ for males and 38,61 and $1 \%$ for females.

A sample of immature lake dwelling trout was collected in january in the pelagic area of Lake Leman. The sex ratio was in favour of females. The percentage of fish aged of two years or three years and more is similar for both sexes being respectively $22 \%$ and $78 \%$ for males and $24 \%$ and $76 \%$ for females. In the sample the percentage of type 1, 2 or 3 trouts was respectively $44,52,4 \%$ with only small differences between sexes.

In the studied tributaries the migration for reproduction generally occured at the end of autumn-beginning of winter and spates act as a favourishing factor. Maximal spawning activity of lake dwelling trout occured between the beginning of december and the beginning of january. The reproduction of the lake dwelling trout is generally a bit later than the one of secontary trouts. The redds of lake dwelling trout, in relation with the bigger size of spawners, are generally longer, with à larger sized substrate and a higher water depth than the redds of sedentary trouts. The multiple spawners of lake dwelling trout spawn generally in the same tributary. Between two reproductions, they seemed to stay in a large portion of the lake including the mouth of their spawning river. The fry (originating from lake dwelling trout spawners), prefed in hatcheries and restoked in tributaries with an unsaturated carrying capacity can represent an important part of the lake dwelling trout spawning run in the corresponding tributaries. 


\title{
LA TRUITE DE MER (SALMO TRUTTA L.) EN NORMANDIE - PICARDIE
}

\author{
G. EUZENAT (1), Françoise FOURNEL (1) et A. RICHARD (2)
}

(1) Conseil Supérieur de la Pêche - DR n० $1-3$, rue Ste Marie - 60200 COMPIEGNE

(2) Conseil Supérieur de la Pêche - DR no $2-84$, rue de Rennes - 35110 CESSON-SEVIGNE

\begin{abstract}
RÉSUMÉ
Les principaux résultats des recherches menées sur la truite de mer, Salmo trutta L., depuis 10 ans dans le Nord-Ouest sont présentés.

Quatre bassins, parmi les plus fréquentés, ont été plus particulièrement suivis: ceux de la Bresle et de l'Arques en Haute-Normandie/Picardie, de l'Orne et de la Touques en BasseNormandie.
\end{abstract}

Pour l'essentiel, les méthodes d'étude, utilisées, selon les bassins, seules ou en complémentarité, comportent:

- le piègeage des migrants, partiel ou total, à la remontée et/ou à la dévalaison

- le marquage, pratiqué sur les stades jeunes ou adultes (frais ou bécards)

- les enquêtes auprès des pêcheurs, en milieu fluvial et maritime côtier.

Ces travaux ont permis d'aboutir à une bonne connaissance des populations: rythmes migratoires, cycle biologique, caractéristiques biométriques et démographiques et, en HauteNormandie, définition des paramètres fonctionnels grâce au suivi pluri-annuel engagé sur le bassin pilote de la Bresle.

La truite de mer en Normandie-Picardie se caractérise par:

- un fort gabarit : les tailles moyennes des poissons de retour varient, selon les bassins, de 46 à $60 \mathrm{~cm}$ pour des poids de 1,3 à $2,9 \mathrm{~kg}$ (extrêmes: 25 à $90 \mathrm{~cm}-0,2$ à $9 \mathrm{~kg}$ )

- une période de remontée étalée de Mai à Janvier, se scindant en deux vagues séparées par un creux estival

- un cycle court, se déroulant généralement en 3 ou 4 ans

- smoltification précoce : 56 à $87 \%$ de smolts en 1 an

- première reproduction survenant, pour 65 à $85 \%$ des sujets, à l'âge de mer 1 .

- une structure d'âge étalée : l'âge total varie de 2 à 10 ans, l'âge de mer de 0 à 8 ans, du fait de la possibilité de retours précoces et/ou multiples; le groupe d'âge 1 - est dominant sur tous les bassins

- un taux de reproduction élevé liè à la prédominance des femelles ( $F / M=2$ à 2,5$)$ et aux frais successifs (1/4 environ des géniteurs se reproduit au moins deux fois).

Des différences s'observent entre les bassins selon la part prise dans les remontées par les poissons de retour précoce $\left(0_{+}\right.$de mer), en liaison ou non avec la reproduction, ou tardif (après 2 hivers de mer).

Sur le plan fonctionnel, les résultats obtenus sur la Bresle mettent en évidence:

- une survie entre l'œuf et le smolt de l'ordre de 0,15 à $0,30 \%$

- une survie marine minimale de 14 à $20 \%$ (retours à partir de smolts marqués)

- un taux d'exploitation global de $46 \%$ minimum, réalisé à plus de $80 \%$ en secteur maritime

permettant d'assurer en fin de compte le remplacement de la génération parentale.

Les résultats acquis sur la truite de mer au cours de la dernière décennie mettent en lumière une stratégie efficace d'occupation du milieu, à l'origine de populations parfois importantes (effectifs des remontées supérieurs à 1.000 individus sur plusieurs des cours d'eau côtiers de la région) ; les mesures de gestion qui en découlent consistent dans les grandes lignes à développer la capacité de production des rivières et à rationaliser l'exploitation pour l'ajuster à la capacité d'accueil. actuelle et future. 


\section{SEA-TROUT, SALMO TRUTTA L., IN NORMANDY-PICARDY}

\section{SUMMARY}

This present paper describes main results of the 10-year study of wild sea-trout, Salmo trutta, in Norman coastal rivers, essentially Bresle, Arques, Touques and Orne ones.

Classic methods are used: trapping of upstream and downstream migrants, branding and Carlin-tagging of smolt and adult fish (fresh and kelt stage), catch survey on river and coast-net fisheries. These different methods are used, independently or not, in the different rivers, but combined in Bresle river_during five consecutive years to study population dynamics.

All fish caught are examined (presence of marks or tags, sea-lices, integrity,...), measured (fork length, maxilla length), weighed end eventually sexed. Scales samples are removed for age determination.

Sea-trout runs virtually in every coastal flowing into the East-Channel, but particularly in Canche, Bresle, Arques, Touques, Dives, Orne and Vire, in looking southwards. most often.

Smolt migration begins in late-February to mid-May, with a peak in the first fortnight of April

Upstream migration occures from may to january, with two distinct runs, separated by a slack period in August-September. In Bresle and Touques rivers, the early run (summer) is always the most important; in Orne river, it is the autumnal run.

Main caracteristics of sea-trout in the North-West of France are:

- a short life-cycle ( 3 or 4 years) with a precocious smoltification ( 56 to $87 \% 1+$ smolts) and a first spawning for 65 to $85 \% 1$, sea-winter fish;

- the great size of adult fish ; mean length and weight range between 46 and $60 \mathrm{~cm}$, and 1,3 and $2,9 \mathrm{~kg}$, depending on the rivers. Finnock runs are generally poor, except in Touques river.

- a wide age-structure : total age range from 2 to 10 years, sea-age from $0_{+}$(finnock) to 8 years (repeat spawners); in all rivers, 1 sea-winter fish are dominant ( 45 to $75 \%$ of the numbers);

- an high reproduction rate, because of the female predominance ( $F / M=2$ to 2,5$)$ and the repeat spawnings ( $25 \%$ of fish, on average, spawn twice at least) ; relative fecundity range between 2120 and $2450 \mathrm{eggs} / \mathrm{kg}$ in Bresle and Orne rivers respectively.

The study on the population dynamics in Bresle river led to the following results:

- 6500 to 8300 smolts are produced each year in relation to initial mean egg deposit of 3700000 eggs; egg-to-smolt survival range between 1,5 and 2,2 smolts in every thousand eggs; it never exceeds $5 \%$

- 14,5 to $20 \%$ of branded smolt return at home river.

- minimum exploitation rate is $46 \%$ ( $39 \%$ by netfishing and $7 \%$ by rod);

- presently, population is self-sustaining, but relation between high escapement and very low egg-to-smolt survival needs to be examined further.

These studies provide clear indications in managing sea-trout fisheries: first, to enhance the carrying capacity of the rivers and secundly, to fit better the fishing effort to the stocks caracteristics.

Future research should focus on the factors governing the reproduction yield, the interactions between residents and migrants and the response of populations to habitat alterations. 


\title{
DIVERSITÉ GÉNÉTIQUE ET GESTION DES POPULATIONS NATURELLES DE TRUITE COMMUNE
}

\author{
R. GUYOMARD
}

INRA-CRJ, Laboratoire de Génétique des Poissons - 78350 JOUY-EN-JOSAS

\begin{abstract}
RÉSUME
Les études de diversité électrophorétique de la truite commune ont confirmé que cette espèce se caractérise, par rapport à d'autres salmonidés, par une diversité génétique élevée $(0,13)$ et un fort degré de différenciation interpopulations représentant plus de $50 \%$ de la variabilité totale. En France, les populations naturelles peuvent être réparties en deux groupes, l'un atlantique, l'autre méditerranéen. Le degré de différenciation entre ceux-ci est comparable à celui observé, chez d'autres salmonidés, entre populations appartenant à des formes identifiées comme des sousespèces différentes. Chacun des deux groupes présente des subdivisions régionales. Par contre, les souches domestiques forment un ensemble très peu différencié, exclusivement issu du groupe atlantique. Aucune différenciation génétique ne permet de distinguer les formes sédentaires et anadromes originaires d'une même rivière; il ne semble donc pas justifié de distinguer, dans la zone atlantique, une entité phylogénétique particulière sur la base du comportement migrateur et d'identifier la truite de mer à une sous-espèce (Salmo trutta trutta). L'existence de flux géniques, consécutifs à des repeuplements, entre stocks domestiques et peuplements naturels est clairement mise en évidence. Les taux d'introgression observés sont très variables et peuvent parfois atteindre $50 \%$. Toutefois, l'impact des repeuplements, exprimée en termes d'efficacité annuelle reste faible dans tous les cas. L'intèrêt de la génétique des populations pour la conservation de la diversité génétique de l'espéce et la gestion des peuplements naturels est également présenté et discuté.
\end{abstract}

\section{GENE DIVERSITY AND MANAGEMENT OF WILD POPULATIONS OF BROWN TROUT}

\section{SUMMARY}

Electrophoretic studies have confirmed that brown trout is one of the most polymorphic (total diversity $=0.13$ ) and differentiated salmonid species (diversity between populations $50 \%$ of the total diversity). A clear-cut dichotomy was found between Atlantic and Mediterranean populations. The amount of differentiation between these two groups is characteristic of morphological subspecies in other salmonid species. Significant gene diversity is present within these two groups. By contrast, the hatchery stocks exhibited little differenciation and originated from the Atlantic area only. No genetic differentiation was observed between anadromous and resident trouts originating from the same river; then, the anadromous form should not be distinguished as a subspecies (Salmo trutta trutta). Gene flows were detected between local populations and hatchery stocks that are releases in the wild. The observed introgression rates reached $50 \%$ in some cases; however, the restocking efficiency always remains low when expressed in terms of annual effects. Finally, we discuss the relevance of these data and population genetics principles to the fishery management and the genetic preservation of brown trout. 


\title{
LA GESTION DES POPULATIONS NATURELLES DE TRUITE COMMUNE EN FRANCE ANALYSÉE DANS UNE PERSPECTIVE HISTORIQUE (1669-1986)
}

\author{
M. THIBAULT \\ Station de Physiologie et d'Ecologie des Poissons, I.N.R.A., \\ 65 , route de St Brieuc - 35042 RENNES Cedex
}

\section{RÉSUMÉ}

Même si le terme de gestion est récent, l'accès à la ressource naturelle renouvelable que constitue la truite commune est codifié à l'échelle nationale depuis le milieu du XVII' siècle. Sont alors mises en place deux mesures qui perdurent jusqu'à nos jours : interdiction de pêche pendant la reproduction et instauration d'une taille au-dessous de laquelle les truites doivent être remises à l'eau.

L'annonce de la redécouverte de la fécondation artificielle de la truite au milieu du XIX $X^{\bullet}$ siècle entraîne l'adoption de mesures supplémentaires qui sont toujours en place, avec un objectif de protection: déversement de juvéniles (œufs puis alevins) destinés à repeupler les cours d'eau; installation de passes à poissons; création de réserves de pêche destinées à protéger le poisson pendant la reproduction; interdiction de vente et de colportage pendant la reproduction.

L'évolution de l'accès à la ressource au cours de la période étudiée se fait au sein de trois grandes tendances qui constituent un bruit de fond prédominant dans les rapports entre la truite et l'homme:

- prééminence des activités économiques liées aux cours d'eau par rapport à la pêche (transport, production d'énergie, élimination des déchets) et des forêts par rapport à la pêche,

- permanence de schémas de pensée anciens dans l'attitude par rapport au poisson, malthusianisme écologique et conception prométhéenne. Ceci se traduit par une restriction croissante de l'accès à la ressource (accroissement de la durée d'interdiction de pêche pendant la reproduction, augmentation de la taille légale et diminution de la pêche professionnelle surtout au cours du $X X^{e}$ siècle) et par un interventionnisme croissant au travers d'une complexification de la réglementation et des déversements de stades jeunes,

- rôle prépondérant des caractéristiques sociales et culturelles liées au poids croissant de la pêche à la ligne depuis la fin du $\mathrm{XIX}^{\mathrm{e}}$ siècle

Même en l'absence de données concernant l'impact des différentes mesures et actions mises en œuvre, il est conclu qu'elles ont eu un effet marginal sur la truite eu égard aux potentialités écologiques de l'espèce et à l'action de l'homme sur l'habitat piscicole.

On s'interroge sur la nécessité de prendre en compte la diversité de l'écologie de l'espèce dans la réglementation et des axes de recherches sont proposés visant à étudier les capacités de régulation et les fluctuations naturelles à l'intérieur d'une population.

\section{SUMMARY}

Even if the term "management" is a recent one, access to natural renewable resources such as the common trout, has been regulated at the national level since the middle of the 17 th century. Two of these measures still exist today, namely, the prohibition of fishing during the reproductive season and the establishing of a minimum fish size, below which trout must be put back into the water.

The rediscovery of artifical fertilisation of trout in the middle of the 19th century resulted in supplementary measures, still observed today, in order to protect the fish. There were: the repopulation of waterways with juvenile fish (eggs and fry), the building of fish passages, the creation of fishing reserves to protect the fish during their reproductive period and prohibition of sale and retailing during the reproductive period.

The development of access to trout in the period studied occured in three main steps:

- the pre-eminence of economic activities both linked to waterways (transport, energy production, waste disposal) and forests with respect to fishing. 
- the persistence of ancient ways of thinking with respect to fish, ecological Malthusianism and Promethean conception. Thus, there was increasing restriction of access to the trout (increase in the period of restriction of fishing during the reproductive period, and increase in the legal size and a decrease in professionnal fishing mainly during the 20th century) and an increase in intervention through the complexity of the reglementation and through the restocking with young stages.

- the influentual role of social and cultural characteristics linked to the increase in rod fishing since the end or the 19 th century.

Even in the absence of data concerning the impact of these different measures, it is concluded that they have had a marginal effect concerning ecological potentiality of the species and the action of man on the fish habitat.

The need to take the ecological diversity of a species into account in regulations is discussed and themes of research are suggested with a view to studying the capacity of regulation and natural fluctuation within a population. 